\title{
Elements of Geometric Stability Theory
}

May 2003

\section{Completeness and quantifier elimination for some classical theories}

We first work out a basic example, with a proof that demonstrates geometroalgebraic, as opposed to syntactical, methods in model theory.

We denote $\mathrm{ACF}_{p}$ the theory of algebraically closed fields of characteristic $p$.

Theorem 1.1 $\mathrm{ACF}_{p}$ is complete and allows quantifier elimination in the language $(+, \cdot, 0,1)$

First we prove

Lemma 1.0.1 (weak form of Steinitz' Theorem) Let $B$ and $C$ of the same uncountable cardinality $\mu$ be in $\mathrm{ACF}_{p}$. Then any isomorphism $\alpha_{0}$ : $B_{0} \rightarrow C_{0}$ between subfields $B_{0} \subseteq B$ and $C_{0} \subseteq C$ of cardinality less than $\mu$ can be extended to an isomorphism $\alpha: B \rightarrow C$.

Proof We enumerate the fields and proceed back-and-forth constructing $\alpha_{i}$ : $B_{i} \rightarrow C_{i}$ of cardinality less than $\mu$.

Suppose $B_{i}$ and $C_{i}$ are isomorphic and of cardinality less than $\mu$. Take the first $b \in B$ not in $B_{i}$. If $b$ is transcendental over $B_{i}$ then by cardinality considerations we can find $c$ transcendental over $C_{i}$. Then

$$
B_{i}(b) \cong B_{i}(x) \cong C_{i}(x) \cong C_{i}(c)
$$


If $b$ is a root of $f_{B}(x)$ over $B_{i}$, irreducible, then the correspondent $f_{C}(x)$ has a root $c$ in $C$ and

$$
B_{i}(b) \cong B_{i}[x] /\left\{f_{B}(x)\right\} \cong C_{i}[x] /\left\{f_{C}(x)\right\} \cong C_{i}[c] .
$$

Corollary 1.1 ACF $F_{p}$ is $\mu$-categorical for $\mu>\aleph_{0}$, and hence complete.

Lemma 1.0.2 For any $A \subseteq F$ and any two $n$-tuples $\bar{b}$ and $\bar{c}$ TFAE:

(i) $q \operatorname{ftp}(\bar{b} / A)=q \operatorname{ftp}(\bar{c} / A)$;

(ii) $\bar{b}$ is conjugated with $\bar{c}$ by an automorphism over $A$;

(iii) $\operatorname{tp}(\bar{b} / A)=\operatorname{tp}(\bar{c} / A)$.

Proof We prove that (i) implies (ii). The rest is obvious.

First consider $n=1$. W.l.o.g. we assume that $A$ is a subfield. If $b$ is transcendental over $A$ then so is $c$ and

$$
A(b) \cong A(x) \cong A(c) \text { over } A .
$$

If $b$ is a root of $f(x)$ over $A$, minimal for $b$, then so is $c$ and

$$
A(b) \cong A[x] /\{f(x)\} \cong A[c] \text { over } A .
$$

If $\bar{b}=\left\langle b_{1} \ldots, b_{n}\right\rangle$ and $q f t p(\bar{b} / A)=q f t p(\bar{c} / A)$ then $q f t p\left(b_{1} / A\right)=q f t p\left(c_{1} / A\right)$ thus by induction there is an isomorphism

$$
\alpha: A\left(b_{1}\right) \rightarrow A\left(c_{1}\right)
$$

Let $\left\langle b_{2}^{\prime} \ldots, b_{n}^{\prime}\right\rangle$ be the image of $\left\langle b_{2} \ldots, b_{n}\right\rangle$ under $\alpha$. Then

$$
q f t p\left(\left\langle c_{1}, b_{2}^{\prime} \ldots, b_{n}^{\prime}\right\rangle / A\right)=q f t p(\bar{b} / A)=q f t p(\bar{c} / A),
$$

hence

$$
\left\langle b_{2}^{\prime} \ldots, b_{n}^{\prime}\right\rangle \text { conj with }\left\langle c_{2}, \ldots, c_{n}\right\rangle \text { over } A c_{1} .
$$

Finally $\bar{b}$ is conjugated with $\bar{c}$ over $A$. 


\section{Proof of QE.}

Let $\varphi(\bar{x})$ be any formula in the field language and

$$
\Phi(\bar{x})=\{\psi(\bar{x}) \text { qfree }: F \models \varphi(x) \rightarrow \psi(\bar{x})\} .
$$

If $\Phi \& \neg \varphi$ is consistent then in monster $F^{\prime} \succ F$ there is a realization $\bar{b}$ of the type. qftp $(\bar{b})$ must be consistent with $\varphi$ for otherwise $\neg \xi(\bar{x})$ is in $\Phi$ for some $\xi \in q f t p(\bar{b})$. Then there exists $\bar{c}$ realizing $q f t p(\bar{b}) \& \varphi$. A contradiction.

Thus $=\Phi \rightarrow \varphi$ and so $\Phi$ is equivalent to its finite part and $\Phi \equiv \varphi$.

Exercise 1.2 Prove a corresponding theorem for the theory of vector spaces.

We also consider the theory of differentially closed fields of characteristic zero $\mathrm{DCF}_{0}$. It is based on the language of fields extended by a symbol of a unary operation D corresponding to a differentiation operator. D obeys the identities

$$
\mathrm{D}(x+y)=\mathrm{D} x+\mathrm{D} y \quad \text { and } \quad \mathrm{D} x y=x \mathrm{D} y+y \mathrm{D} x .
$$

$\mathrm{DCF}_{0}$ is axiomatised as an algebraically closed field with an operator D satisfying (1) and the axiom scheme stating that for any differential polynomials $g(y)$ of order $n>0$ and $f(y)$ of order $m<n$ over the field there is a solution of the system

$$
g(y)=0 \& f(y) \neq 0
$$

Theorem 1.3 $\mathrm{DCF}_{0}$ is complete and allows elimination of quantifiers.

The proof of the theorem (see e.g. $[\mathrm{P}]$ ) is quite similar to the proof of Theorem 1.1. One studies extensions of an isomorphism between differential subfields in a way similar to the proof of Lemma 1.0.1 and then uses the back-and-forth method to establish an elementary equivalence between two models of $\mathrm{DCF}_{0}$ and to prove QE.

We also want to consider as one of our basic examples the structure on the complex numbers

$$
\mathbb{C}^{e}=\left(\mathbb{C},+, P^{(3)}\right) \text {, where } P^{(3)}(x, y, z) \equiv e^{x}+e^{y}=e^{z} .
$$


Notice that the subgroup $2 \pi i \mathbb{Z}$ is definable in $\mathbb{C}^{e}$ as

$$
\left\{v \in \mathbb{C}: \forall x, y, z \quad e^{x}+e^{y}=e^{z} \leftrightarrow e^{x}+e^{y}=e^{z+v}\right\} .
$$

Now, if we introduce a definable set $\mathbb{C}^{*}=\mathbb{C} / 2 \pi i \mathbb{Z}$ and a definable canonical homomorphism exp : $\mathbb{C} \rightarrow \mathbb{C}^{*}$ we get an equivalent representation of the structure as a two-sorted structure $\left(\mathbb{C}, \mathbb{C}^{*} \cup\{0\}\right)$ with the additive group structure $(\mathbb{C},+)$ on the first sort, the field structure $\left(\mathbb{C}^{*} \cup\{0\}, \cdot,+\right)$ on the second sort and exp mapping the first sort into the second sort.

To capture the most interesting properties of this structure it is not enough to consider its first-order theory only. A more relevant language is the language $L_{\omega_{1}, \omega}$ allowing conjunctions and disjunctions over countable sets of formulas with finite strings of variables.

We state without proof (which can be found in [Z1])

Theorem 1.4 A natural $L_{\omega_{1}, \omega}$-sentence axiomatising $\mathbb{C}^{e}$ has models and is categorical in all uncountable cardinalities.

Any automorphism of the field on the second sort $\mathbb{C}^{*} \cup\{0\}$ can be lifted to an automorphism of the structure $\mathbb{C}^{e}$.

\section{Closure operators in abstract structures}

\subsection{Algebraic closure}

Definition Given $A \subseteq M$, a formula $\varphi(x)$ with parameters in $A$ is called algebraic if for some $m>0$

$$
M \models \exists^{=m} x \varphi(x) .
$$

A type over $A$ containing an algebraic formula is said to be algebraic.

$\operatorname{acl}(A)=\{b \in M:$ there is an algebraic $\varphi(v)$ over $A$ such that $M \models \varphi(b)\}$.

Lemma 2.1.1 The following properties of acl hold in any structure:

$$
\begin{gathered}
A \subseteq \operatorname{acl}(A) \\
A \subseteq B \Rightarrow \operatorname{acl}(A) \subseteq \operatorname{acl}(B) \\
\operatorname{acl}(\operatorname{acl}(A))=\operatorname{acl}(A) .
\end{gathered}
$$




\section{Proof Easy. $\square$}

Remark For any field $F$ and $A \subseteq F$, letting the field theoretic algebraic closure of $A$ to be $\operatorname{Acl}(A)$, we have

$$
\operatorname{Acl}(A) \supseteq \operatorname{acl}(A)
$$

By elimination of quantifiers, in algebraically closed fields we have

$$
\operatorname{Acl}(A)=\operatorname{acl}(A) .
$$

We hence have a classical

Corollary 2.1 If $b$ and $c$ are algebraic over $A$ in a field $F$, then so are $b+c$ and bc.

Definition An elementary monomorphism $\alpha: A \rightarrow M^{\prime}$, for $A \subseteq M$ and $M, M^{\prime}$ structures in the same language $L$, is an injective map which preserves $L$-formulas. An elementary monomorphism which is a bijection of $A \subseteq M$ onto $A^{\prime} \subseteq M^{\prime}$ is called an elementary isomorphism between $A$ and $A^{\prime}$.

Proposition 2.1 (Uniqueness of closure) Any elementary isomorphism $\alpha$ between $A \subseteq M$ and $A^{\prime} \subseteq M^{\prime}$ can be extended to $\operatorname{acl}(A) \rightarrow \operatorname{acl}\left(A^{\prime}\right)$.

Proof Let $B=\operatorname{acl}(A)$ and $B^{\prime}=\operatorname{acl}\left(A^{\prime}\right)$. Enumerate $B=\left\{b_{i}: i<\mu\right\}$, $\mu=$ card $B$, and go by transfinite induction extending $\alpha$ to $A \cup\left\{b_{i}: i<\gamma\right\}$, finding for $b_{\gamma}$ a corresponding element $b_{\gamma}^{\prime} \in B^{\prime}$ such that

$$
A \cup\left\{b_{i}: i \leq \gamma\right\} \mapsto A^{\prime} \cup\left\{b_{i}^{\prime}: i \leq \gamma\right\}
$$

is an elementary isomorphism. In order to see this is possible notice that the type

$$
p_{\gamma}=\operatorname{tp}\left(b_{\gamma} /\left(A \cup\left\{b_{i}: i<\gamma\right\}\right)\right)
$$

is algebraic by definition, and thus is principal. Hence the type $p_{\gamma}^{\alpha}$ obtained by replacing parameters in $p_{\gamma}$ by their images under $\alpha$ is also algebraic and principal. Hence $p_{\gamma}^{\alpha}$ is realised by some element of $M^{\prime}$ which we can take for 
$b_{\gamma}^{\prime}$. Notice also that before reaching $\gamma=\mu$ we list all elements of $p_{\gamma}$ as some $b_{i_{1}}, \ldots b_{i_{n}}$, and hence we get all elements of $p_{\gamma}^{\alpha}$ as some $b_{i_{1}}^{\prime}, \ldots, b_{i_{n}}$.

It follows that by reaching step $\mu$ we have $B$ as a domain of $\alpha$ and, since all the realisations of algebraic types over $A^{\prime}$ are in $B^{\prime}$, we have $\alpha(B)=B^{\prime}$.

\section{$2.2 \lambda$-closure}

We now generalise slightly the definition of algebraic closure.

Definition Given an infinite regular cardinal $\lambda$, a structure $M$ of cardinality at least $\lambda$ and $A \subseteq M$ we say that a formula $\varphi(x)$ over $A$ is $\lambda$-small in $M$ if card $\varphi(M)<\lambda$.

The $\lambda$-closure of $A$ in $M$ is defined as

$$
\operatorname{cl}^{\lambda}(A)=\bigcup\{\varphi(M): \varphi(x) \text { is a } \lambda \text {-small formula over } A\} .
$$

We say that $\lambda$-smallness is weakly definable in $M$ if for any finite $\bar{a}$ in $M$ and $b \in \operatorname{cl}^{\lambda}(\bar{a})$ there exist formulas $\varphi(x, \bar{y})$ and $\exists^{<\lambda} x \varphi(x, \bar{y})(\bar{y}$ the only free variables in $\left.\exists^{<\lambda} x \varphi(x, \bar{y})\right)$ without parameters such that $\varphi(b, \bar{a})$ and $\exists^{<\lambda} x \varphi(x, \bar{a})$ hold in $M$, and for any $\bar{a}^{\prime}$ in $M$

$$
M \vDash \exists^{<\lambda} x \varphi\left(x, \bar{a}^{\prime}\right) \Rightarrow \operatorname{card} \varphi\left(M, \bar{a}^{\prime}\right)<\lambda .
$$

Example It follows from Theorem 1.4, second clause, that in the structure $\mathbb{C}^{e}$, for $\lambda=\omega_{1}$, the $\lambda$-closure for $A \subseteq \mathbb{C}$ is exactly

$$
\operatorname{cl}^{\lambda}(A)=\ln (\operatorname{Acl}(\exp A)) \text {, where } \ln X=\{z \in \mathbb{C}: \exp z \in X\} .
$$

Hence we deduce that in $\mathbb{C}^{e}$

$$
\begin{aligned}
& b \in \operatorname{cl}(A) \text { iff } b \in \varphi(\mathbb{C}) \text { for some } A \text {-definable analytic } \\
& \text { set } \varphi(\mathbb{C}) \text { of }(\text { complex analytic) dimension } 0 .
\end{aligned}
$$

Also, $\omega_{1}$-smallness is weakly definable in $\mathbb{C}^{e}$ by Theorem 1.4. 
Lemma 2.2.1 The following properties of $\mathrm{cl}=\mathrm{cl}^{\lambda}$ hold in any structure:

$$
\begin{gathered}
A \subseteq \operatorname{cl}(A) ; \\
A \subseteq B \Rightarrow \operatorname{cl}(A) \subseteq \operatorname{cl}(B) ;
\end{gathered}
$$

If also $\lambda$-smallness is weakly definable in the structure then

$$
\operatorname{cl}(\operatorname{cl}(A))=\operatorname{cl}(A)
$$

Proof The first two are obvious.

To prove (7) suppose $B \subseteq \operatorname{cl}(A)$ and $c \in \operatorname{cl}(B)$. We may assume that $B=$ $\left\{b_{1}, \ldots, b_{m}\right\}$. We then have $\vDash \psi_{i}\left(b_{i}\right)$ for some $\lambda$-small $A$-definable $\psi_{i}(y)$ and $\phi(x, \bar{b})$ for some $\lambda$-small $\phi(x, \bar{b})$ such that $\phi(x, \bar{y})$ is $A$-definable. We obviously have

$$
M \vDash \exists \bar{y} \phi(c, \bar{y}) \& \exists^{<\lambda} x \phi(x, \bar{y}) \& \bigwedge_{i} \psi_{i}\left(y_{i}\right)
$$

and, on the other hand, the $A$-definable set

$$
\bigcup\left\{\phi\left(M, b_{1}^{\prime}, \ldots, b_{m}^{\prime}\right): \& \exists^{<\lambda} x \phi\left(x, b_{1}^{\prime}, \ldots, b_{m}^{\prime}\right) \& \bigwedge_{i} \psi\left(b_{i}^{\prime}\right)\right\}
$$

is of cardinality less than $\lambda$.

Remark The Uniqueness property for $\mathrm{cl}^{\lambda}$ ( Proposition 2.1) does not hold in general, and does not hold in $\mathbb{C}^{e}$ in particular.

\subsection{Closure in differentially closed fields}

This is a new type of closure which anyway is linked to a notion of smallness given in general by model theoretic means.

Definition Given a differential field $F$ and its subfield $A$ we say that $b \in F$ is differential-algebraic over $A$ if $b$ is a solution of a non-trivial differential equation $f(y)=0$ over $A$.

Define $\operatorname{Dfcl}(A)$ to be the set of all differential algebraic elements over $A$ in a given model of $\mathrm{DCF}_{0}$. 
Lemma 2.3.1 Given a differentially closed field $F$ and letting $\mathrm{cl}=\mathrm{Dfcl}$ we have that (5),(6) and (7) hold in F.

Idea of Proof (5) and (6) are obvious. We discuss the proof of (7). One uses a dimension theory based on Morley rank. Assuming that the field $C$ of constants of $F$ (i.e. $c \in F$ such that $\mathrm{D} c=0$ ) is of dimension 1 we see $F$ as an infinite dimensional object. Correspondingly the space of solutions of a differential equation $f(y)=0$ of order $m$ (that is $\mathrm{D}^{m} y$ is the maximal differential occurring in $f$ ) is of dimension $m$, so is small. Hence, if $b$ is a solution of a differential equation $g(y)=0$ with coefficients $a_{1}, \ldots, a_{n}$ which are differential-algebraic over some $A$, then $b$ belongs to a finite-dimensional space (of dimension depending on the order of $g$ and the orders of the corresponding equations for $\left.a_{1}, \ldots a_{n}\right)$. This space, by elimination of quantifiers, is defined by an equation over $A$, and this proves that $b \in \operatorname{Dfcl}(A)$.

Remark Uniqueness of closure holds for Dfcl in the following form

Given $A \subseteq F, F$ a model of $\mathrm{DCF}_{0}$, there is a unique up to isomorphism $\mathrm{DCF}_{0}$-model $F(A)$, prime over $A$, and

$$
F(A)=\operatorname{Dfcl}_{F(A)}(A)
$$

The reasons that $F(A)$ can be constructed in a unique way are similar to those used in the proof of Proposition 2.1. Namely, going by a similar inductive process we choose $b_{\gamma}$ so that $p_{\gamma}$ is of minimal Morley rank, then $p_{\gamma}$ turns out to be principal.

\section{$3 \quad$ Minimal structures}

For a structure $M$ with a closure operator cl the exchange principle states that

$$
\text { For any } A \subseteq M, b, c \in M: \quad b \in \operatorname{cl}(A, c) \backslash \operatorname{cl}(A) \Rightarrow c \in \operatorname{cl}(A, b) \text {. }
$$


Definition A structure $M$ is said to be minimal if any definable (using parameters) subset of $M$ is either finite or a complement of a finite.

Lemma 3.0.2 In minimal structures, letting $\mathrm{cl}=\mathrm{acl}$, the exchange principle holds.

Proof Suppose $b \in \operatorname{acl}(A, c) \backslash \operatorname{acl}(A)$. Then for some $\varphi(x, y)$ over $A$ and some $m$

$$
M \models \varphi(b, c) \& \exists^{\leq m} x \varphi(x, c) .
$$

W.l.o.g. we assume

$$
M \models \varphi(x, y) \rightarrow \exists^{\leq m} x \varphi(x, y) .
$$

Suppose, towards a contradiction, that $\varphi(b, M)$ is infinite. Then card $(\neg \varphi(b, M)) \leq k$ for some $k$, i.e. $M \models \exists \leq k y \neg \varphi(b, y)$ and

$$
B=\left\{b^{\prime} \in M: M \models \exists \leq k y \neg \varphi\left(b^{\prime}, y\right)\right\}
$$

is infinite, since $b \notin \operatorname{acl}(A)$. Choose distinct $b_{1}, \ldots, b_{m+1} \in B$. Then

$$
\varphi\left(b_{1}, M\right) \cap \cdots \cap \varphi\left(b_{m+1}, M\right)
$$

is infinite and thus contains a point $c^{\prime}$. It contradicts $M \vDash \exists \leq m x \varphi\left(x, c^{\prime}\right)$.

Again, we would like to generalise the notion of a minimal structure.

Definition A structure $M$ is said to be $\lambda$-minimal if card $M \geq \lambda$ and any definable subset of $M$ is either $\lambda$-small or a complement of a $\lambda$-small subset.

We say that a formula $\phi(y, z)$ has the strict $\lambda$-order property in $M$ if $\phi(y, z)$ defines on $M$ a partial order (transitive but not necessarily antisymmetric) with a chain of ordinal type $\lambda$.

Example $\mathbb{C}^{e}$ is $\lambda$-minimal, by section 2.2 .

Lemma 3.0.3 Suppose $M$ is $\lambda$-minimal and $\lambda$-smallness is weakly definable in $M$. Then the exchange principle holds for $\mathrm{cl}^{\lambda}$, or card $M=\lambda$ and $M$ has the strict $\lambda$-order property. 
Proof We follow the proof of Lemma 3.0.2. Below cl stands for $\mathrm{cl}^{\lambda}$.

Suppose $b \in \operatorname{cl}(A, c) \backslash \operatorname{cl}(A)$. Then for some $\varphi(x, y)$ over $A$ and some $m$

$$
M \models \varphi(b, c) \& \exists^{<\lambda} x \varphi(x, c) .
$$

By letting $\varphi(x, y):=\varphi(x, y) \& \exists^{<\lambda} x \varphi(x, y)$ we assume

$$
M \vDash \varphi(x, y) \rightarrow \exists^{<\lambda} x \varphi(x, y) .
$$

If card $\varphi(b, M)<\lambda$ then $c \in \operatorname{cl}(A, b)$, in correspondence with the exchange principle.

Suppose that the exchange principle does not hold in $M$. Then, for some $b, c$ and $A$ as above, card $\varphi(b, M) \geq \lambda$. Then card $\neg \varphi(b, M)<\lambda$ and

$$
b \in B=\left\{b^{\prime} \in M: M \models \exists^{<\lambda} y \neg \varphi\left(b^{\prime}, y\right)\right\}
$$

is of cardinality at least $\lambda$, since $b \notin \operatorname{cl}(A)$.

Now, let $\left\{b_{i}: i<\lambda\right\} \subseteq B$ be a $\lambda$-sequence of distinct elements of $B$. For any ordinal $\alpha<\lambda$

$$
\operatorname{card}\left(\bigcup_{i<\alpha} \neg \varphi\left(b_{i}, M\right)\right)<\lambda \text {, }
$$

hence

$$
\bigcap_{i<\alpha} \varphi\left(b_{i}, M\right) \neq \emptyset
$$

and thus the set contains a point, say $c_{\alpha}$. We have correspondingly

$$
\left\{b_{i}: i<\alpha\right\} \subseteq \varphi\left(M, c_{\alpha}\right) .
$$

If card $M>\lambda$ then card $B=$ card $M>\lambda$ and we can find $c_{\lambda} \in B$ such that $\left\{b_{i}: i<\lambda\right\} \subseteq \varphi\left(M, c_{\lambda}\right)$, contradicting the definition of $B$. So card $M=$ card $B$ has to be $\lambda$. In particular, we may assume $\left\{b_{i}: i<\lambda\right\}=B$.

Let $x_{1} \prec x_{2}$ denote the formula stating that

$$
B \cap \varphi\left(x_{1}, M\right) \subseteq B \cap \varphi\left(x_{2}, M\right) .
$$

Obviously this defines a partial order. Suppose $c_{1} \prec \cdots \prec c_{i} \ldots$ is a chain in the partial order of ordinal type $\alpha$, for some $\alpha<\lambda$. We have then that

$$
B_{\alpha}=\bigcup_{i<\alpha} B \cap \varphi\left(c_{i}, M\right)
$$


is of cardinality less than $\lambda$. Let $b_{\alpha}$ be an element in $M \backslash B_{\alpha}$, then by above there is $c_{\alpha} \in M$ such that $B_{\alpha} \cup\left\{b_{\alpha}\right\} \subseteq \varphi\left(c_{\alpha}, M\right)$. We then have $c_{i} \prec c_{\alpha}$ for all $i<\alpha$, which means that the $\alpha$-chain is not maximal and can be extended to a chain of ordinal type $\lambda$.

Example Let $\lambda>\aleph_{0}$ be a cardinal. Consider the structure $\left(M_{\lambda},<\right)$ with $M_{\lambda}=\lambda \times \mathbb{Q}$ and $<$ the lexicographic order on the set. Then $M_{\lambda}$ is densely ordered without endpoints. This is known to have elimination of quantifiers and so any definable subset $S$ of $M_{\lambda}$ is a union of finitely many intervals and points. If one of the intervals is given by $x>a$ then card $S=$ card $M=\lambda$. Otherwise card $S<\lambda$. Hence $M_{\lambda}$ is $\lambda$-minimal, but it does not obey the exchange principle.

\subsection{The pregeometry and the geometry of a minimal structure.}

Definition An [abstract] pregeometry is a set $M$ with an operator

$$
\mathrm{cl}: 2^{M} \rightarrow 2^{M}
$$

such that, for any $A \subseteq M, \operatorname{cl}(A)=\left\{\operatorname{cl}\left(A^{\prime}\right): A^{\prime} \subseteq A\right.$ finite $\}$ and conditions (5)-(8) above are satisfied.

A pregeometry is said to be a geometry if

$$
\text { for any } a \in M \quad \operatorname{cl}(\{a\})=\{a\}
$$

\section{Getting a geometry from a pregeometry}

Lemma 3.1.1 The relation $\sim$ on $M \backslash \operatorname{cl}(\emptyset)$ defined as

$$
x \sim y \text { iff } \operatorname{cl}(x)=\operatorname{cl}(y)
$$

is an equivalence relation. 
Proof Follows from the exchange principle.

Definition For a pregeometry $M$ define the set

$$
\hat{M}=(M \backslash \operatorname{cl} \emptyset) / \sim
$$

(quotient under the equivalence relation $\sim$.)

Then any point in $\hat{M}$ is of the form $\hat{a}=\operatorname{cl}\{a\} \backslash \mathrm{cl} \emptyset$, for a corresponding $a \in M \backslash \mathrm{cl}$

emptyset. For a subset $\hat{A}=\{\hat{a}: a \in A\} \subseteq \hat{M}$ define

$$
\operatorname{cl}(\hat{A})=\{\hat{b}: b \in \operatorname{cl}(A)\} .
$$

The operator cl on $\hat{M}$ satisfies then (5)-(9) and thus $\hat{M}$ is a geometry.

Definition Given a subset $D \subseteq M$ of a pregeometry we construct a pregeometry $M_{D}$, the localisation of $M$ with respect to $D$ : the set of $M_{D}$ is just $M$ and $\operatorname{cl}_{D}(A)=\operatorname{cl}(D \cup A)$.

Subspaces of a pregeometry are subset of the form $\operatorname{cl}(A)$. Pregeometry is said to be locally finite if $\operatorname{cl}(A)$ is finite whenever $A$ is.

Example Vector spaces over division rings are pregeometries if we let

$$
\operatorname{cl}(A)=\operatorname{span}(A) .
$$

The projective space associated with a vector space $M$ is defined exactly as the geometry $\hat{M}$.

The affine space associated with a vector space $M$ is defined on the same set $M$ by the new closure-operator:

$$
\operatorname{cl}_{\mathrm{aff}}(A)=A+\operatorname{span}(A-A)
$$

where $A-A=\left\{a_{1}-a_{2}: a_{1}, a_{2} \in A\right\}$.

Exercise Show that an affine space is a geometry and its localisation with respect to any point is isomorphic to the initial vector space pregeometry. 
Definition $\mathrm{A}$ set $A$ is said to be independent if $\operatorname{cl}(A) \neq \operatorname{cl}\left(A^{\prime}\right)$ for any proper subset $A^{\prime} \subset A$.

A maximal independent subset of a set $A$ is said to be a basis of $A$.

Lemma 3.1.2 Any two bases $B$ and $C$ of a set $A$ are of the same cardinality.

Proof First consider the case when, say $B$, is finite and consists of $n$ elements $b_{1}, \ldots b_{n}$. There exists $c \in C$ such that

$$
c \in \operatorname{cl}\left(b_{1}, \ldots b_{n}\right) \backslash \operatorname{cl}\left(b_{1}, \ldots b_{n-1}\right),
$$

for otherwise $B$ is not independent. By the exchange principle $\left\{c, b_{1}, \ldots b_{n-1}\right\}$ is a basis of $A$. In the localisation $M_{c}$ sets $\left\{b_{1}, \ldots b_{n-1}\right\}$ and $C \backslash\{c\}$ are bases of $A$. By induction on $n$ the statement follows.

Consider now the case when both $B$ and $C$ are infinite. It follows from the finite character of cl that for any $b \in B$ there is a minimal finite $C_{b} \subset C$ such that $b \in \operatorname{cl}\left(C_{b}\right)$. Thus there is a mapping of $B$ into $\mathcal{P}_{\text {fin }}(C)$, the set of all finite subsets of $C$. The mapping is finite-to-one, since by the above analysis of the finite dimensional case the set

$$
\left\{d \in B: C_{d}=C_{b}\right\}
$$

is an independent subset of $\operatorname{cl}\left(C_{b}\right)$ and its size is not bigger than the size of $C_{b}$.

It follows card $B \leq$ card $C$. By symmetry card $B=$ card $C$.

Now we can give

Definition For any subset $A$ of a pregeometry define the $\operatorname{dimension} \operatorname{dim} A$ to be the cardinality of a basis of $A$. If also $B \subseteq A$ then $\operatorname{dim}(A / B)$ is the dimension of $A$ in pregeometry $M_{B}$.

Lemma 3.1.3 (the addition formula) For any $B \subseteq A \subseteq M$

$$
\operatorname{dim}(A / B)+\operatorname{dim}(B)=\operatorname{dim}(A) .
$$


Proof One can construct a basis of $A$ by adjoining a basis of $A$ in $M_{B}$ to a basis of $B$ in $M$.

Examples The transcendence degree tr.deg. $A$ of a subset $A$ of an algebraically closed field $F$ is defined as $\operatorname{dim} A$ in the above sense with $\mathrm{cl}=\mathrm{Acl}$. Since any field is a subfield of an algebraically closed one, the definition is applicable for subsets of any field.

Lemma 3.1.4 For $X, Y \subseteq M$, subspaces of a pregometry,

$$
\operatorname{dim}(X \cup Y) \leq \operatorname{dim} X+\operatorname{dim} Y-\operatorname{dim}(X \cap Y) .
$$

Proof Let $Z$ be a basis of $X \cap Y$. Let $Z \cup X_{0}$ and $Z \cup Y_{0}$ be bases of $X$ and $Y$, correspondingly. Then $\operatorname{cl}\left(X_{0} \cup Z \cup Y_{0}\right)=\operatorname{cl}(X \cup Y)$ and thus $\operatorname{dim}(X \cup Y) \leq\left|X_{0} \cup Z\right|+\left|Z \cup Y_{0}\right|-|Z|$.

\section{Homogeneity}

Definition A subset $A$ of a structure $M$ is said to be indiscernible over $B$ if $t p(\bar{a} / B)=\operatorname{tp}\left(\bar{a}^{\prime} / B\right)$ for any two $n$-tuples of distinct elements of $A$ for any finite $n$.

Proposition 3.1 Let $M$ be a $\lambda$-minimal structure with $\lambda$-smallness weakly definable, $\mathrm{cl}=\mathrm{cl}^{\lambda}, A, B \subseteq M$ and $A$ independent over $B$. Then $A$ is indiscernible over $B$.

Proof Consider $\bar{a}=\left\langle a_{1}, \ldots a_{n}\right\rangle, \quad \bar{a}^{\prime}=\left\langle a_{1}^{\prime}, \ldots, a_{n}^{\prime}\right\rangle$ all with distinct coordinates from $A$. In case the size $n=1 \operatorname{tp}(a / B)$ is just the set of those formulas $\varphi(x)$ over $B$ which have card $\varphi(M) \geq \lambda$. The same characterises $\operatorname{tp}\left(a^{\prime} / B\right)$. Thus the types are equal.

For $n>1$ suppose, as an inductive hypothesis, the tuples have the same type over $B$. Then for $a_{n+1} \in A \backslash\left\{a_{1}, \ldots a_{n}\right\}$ and any formula $\varphi(\bar{x}, y)$ over $B$

$$
M \models \varphi\left(\bar{a}, a_{n+1}\right) \text { iff card } \varphi(\bar{a}, M) \geq \lambda \text { iff } M \models \exists^{<\lambda} x \neg \varphi(\bar{a}, x) .
$$


Since $\operatorname{tp}(\bar{a} / B)=\operatorname{tp}\left(\bar{a}^{\prime} / B\right)$ we have card $\varphi\left(\bar{a}^{\prime}, M\right) \geq \lambda$ which yields $M \models \varphi\left(\bar{a}^{\prime}, a_{n+1}^{\prime}\right)$ for any $a_{n+1}^{\prime} \in A$, distinct from the coordinates of $\bar{a}^{\prime} . \square$

Corollary 3.1 Any subset $A$ independent over $B$ in a minimal structure $M$ (with $\mathrm{cl}=\mathrm{acl}$ ) is indiscernible over $B$.

Definition A structure $M$ is said to be homogeneous if given subsets $B, B^{\prime} \subseteq M$ of cardinality less that card $M$ and an elementary isomorphism $\alpha: B \rightarrow B^{\prime}$ there is an extension of $\alpha$ to an automorphism of $M$.

Definition Given a structure $M$ with a closure operator cl we say that $M$ satisfies the countable closure property if $\mathrm{cl}(X)$ is at most countable for any finite $X \subseteq M$.

Remark It is obvious that a structure in a countable language has the countable closure property for $\mathrm{cl}=\mathrm{acl}$ and indeed for $\mathrm{cl}=\mathrm{cl}^{\aleph_{1}}$.

Lemma 3.1.5 Any minimal structure with a countable closure property is homogeneous.

Proof Suppose $B, B^{\prime} \subseteq M$ are of cardinality less than card $M$ and there is an elementary monomorphism $\alpha: B \rightarrow B^{\prime}$. It follows $\operatorname{dim} B=\operatorname{dim} B^{\prime}$.

By the addition formula $\operatorname{dim} M / B=\operatorname{dim} M / B^{\prime}$ or $\operatorname{dim} B=\operatorname{dim} M \geq \aleph_{0}$. The latter contradicts the cardinality assumptions since in this case card $B \geq$ $\operatorname{dim} B=\operatorname{card} M$.

Let $A$ and $A^{\prime}$ be bases of $M$ over $B$ and $B^{\prime}$ correspondingly. Since

$$
\text { card } A=\operatorname{card} A^{\prime}
$$

there is a bijection $\beta: A \cup B \rightarrow A^{\prime} \cup B^{\prime}$ extending $\alpha$. By Corollary $3.1 \beta$ is elementary. Proposition 2.1 finishes the proof. $\square$

Remark Homogeneity is very important in the study of pregeometries. The uniqueness of closure (Proposition 2.1) is essential for the proof of homogeneity, but often weaker versions suffice. E.g. if $F(A)$ is a differentially 
closed field of characteristic zero constructed as a prime model over a Dfclindependent subset $A$ (see the remark in the end of section 2) then basically the same argument as above yields homogeneity of $M$.

On the other hand it is possible to work effectively with a weaker version of homogeneity.

Definition $M$ is said to be $\aleph_{0}$-homogeneous if for every two $n$-tuples $\bar{a}$ and $\bar{a}^{\prime}$ in $M$ of equal types over $\emptyset$ and any $b \in M$ there is a $b^{\prime} \in M$ such that

$$
\operatorname{tp}(\bar{a} b / \emptyset)=\operatorname{tp}\left(\bar{a}^{\prime} b^{\prime} / \emptyset\right)
$$

$M$ is said to be $\aleph_{0}$-homogeneous over submodels, if it is $\aleph_{0}$-homogeneous and for every two $n$-tuples $\bar{a}$ and $\bar{a}^{\prime}$ in $M$ and any $M_{0} \prec M$, card $M_{0}<$ card $M$, such that

$$
\operatorname{tp}\left(\bar{a} / M_{0}\right)=\operatorname{tp}\left(\bar{a}^{\prime} / M_{0}\right)
$$

and any $b \in M$ there is $b^{\prime} \in M$ satisfying

$$
\operatorname{tp}\left(\bar{a} b / M_{0}\right)=\operatorname{tp}\left(\bar{a}^{\prime} b^{\prime} / M_{0}\right) .
$$

Exercise 3.1 If $M$ is countable and $\aleph_{0}$-homogeneous then $M$ is homogeneous.

Exercise 3.2 If $M$ is $\aleph_{1}$-minimal, satisfies the exchange principle for $\mathrm{cl}^{\aleph_{1}}$, card $M=\aleph_{1}$ and $M$ is $\aleph_{0}$-homogeneous over submodels then any bijection between two bases of $M$ can be extended to an automorphism of $M$.

Example $\mathbb{C}^{e}$ is not homogeneous but is $\aleph_{0}$-homogeneous over submodels. Moreover, any elementary substructure of $\mathbb{C}^{e}$ of cardinality $\aleph_{1}$ satisfies the conditions of Exercise 3.2. Under GCH this holds for $\mathbb{C}^{e}$ itself. 


\section{Dimension notion in strongly minimal struc- tures}

\subsection{Strong minimality}

Theorem 4.1 Minimal structures of infinite dimension are $\aleph_{0}$-saturated.

Proof Let $\varphi(x)$ be a formula over a finite $A$. Either $\varphi(M)$ is finite, or $M \backslash \operatorname{acl}(A) \subseteq \varphi(M)$. Thus for any type $p$ over $A$

$$
p(M)=\bigcap_{\varphi \in p} \varphi(M)
$$

either contains the nonempty set $M \backslash \operatorname{acl}(A)$ or is a nonempty subset of some finite $\varphi_{i}(M)$.

Remark If $M$ has the countable closure property then the same argument proves that $M$ is saturated.

Definition We say finiteness is strongly definable in $M$ if for every formula $\varphi(x, \bar{y})$ there is a number $m_{\varphi}$ such that for any $\bar{a}$ in $M$

$$
\operatorname{card} \varphi(M)>m_{\varphi} \Rightarrow \operatorname{card} \varphi(M) \geq \aleph_{0} \text {. }
$$

This is in fact a weak version of the negation of the finite cover property often referred to as not f.c.p.

Remark The property defined above implies that for every $\varphi(x, \bar{y})$ we can state by a first order formula that for any $\bar{a}$ both $\varphi(M, \bar{a})$ and $\neg \varphi(M, \bar{a})$ are infinite.

Remark One can naturally extend the definition to say $\lambda$-smallness is strongly definable in $M$.

Lemma 4.1.1 Finiteness is strongly definable in any minimal structure of infinite dimension. 
Proof Suppose towards a contradiction that $\varphi(x, \bar{y})$ is a counterexample. Then for any $m$ there is a $\bar{a}_{m}$ in $M$ such that $\varphi\left(M, \bar{a}_{m}\right)$ is finite of size bigger than $m$. Then its complement is infinite. Hence $\bar{a}_{m}$ realises the formula

$$
\psi_{m}(\bar{y}):=\exists^{>m} x \varphi(x, \bar{y}) \& \exists^{>m} x \neg \varphi(x, \bar{y}) .
$$

It follows that the type $p=\left\{\psi_{m}: m \in \mathbb{N}\right\}$ is consistent. By $\aleph_{0}$-saturatedness there is a realisation $\bar{a}$ of $p$ in $M$. By construction both $\varphi(M, \bar{a})$ and $\neg \varphi(M, \bar{a})$ are infinite. The contradiction.

Corollary 4.1 Any structure elementary equivalent to a minimal structure of infinite dimension is minimal too.

Definition A minimal structure is said to be strongly minimal (abbreviated s.m.) if it is elementarily equivalent to a minimal structure of infinite dimension.

An important property of a s.m. structure $M$ is that its minimality is first order, i.e. the theory $\operatorname{Th}(M)$ of $M$ forces every model of it to be minimal. Moreover, we have

Lemma 4.1.2 If $M$ is minimal, $A \subseteq M$ and $N=\operatorname{acl}(A)$ an infinite substructure, then $N \preccurlyeq M$.

Proof By Tarski's criterion we need to check that if a formula $\varphi(x)$ over $N$ has a realisation $b$ in $M$ then it has one in $N$. If $\varphi(M)$ is finite then it is a subset of $N$ by the properties of closure. If $\varphi(M)$ is infinite then $\neg \varphi(M)$ is finite and so $\varphi(M) \cap N \neq \emptyset$.

Remark The lemma has an obvious generalisation to $\lambda$-minimal structures.

Corollary 4.2 If an infinite dimensional $M$ is minimal and has a countable closure property then $\operatorname{Th}(M)$ is categorical in any uncountable cardinality, in particular it has a model in any such cardinality independently on the cardinality of the language. 


\subsection{Rank notion for sets definable in s.m. structures.}

We assume below $M$ is minimal of infinite dimension. To any definable subset $S$ in $M$ we attach a number called the (Morley) rank of $S$. This in fact is a dimension notion dual to the dimension introduced above. It measures the dimension of macrosets $S \subseteq M^{n}$ in contrast with the dimension of microsets $\bar{s} \in M^{n}$.

Definition Let $A \subseteq M$ be finite. For an $A$-definable subset $S \subseteq M^{n}$ let the rank of $S$ to be

$$
\operatorname{rk} S=\max _{\left\langle s_{1}, \ldots s_{n}\right\rangle \in S} \operatorname{dim}\left(\left\{s_{1}, \ldots s_{n}\right\} / A\right)
$$

Remark The rank notion coincides with the notion of Morley rank.

Lemma 4.2.1 (i) rk $\varphi(M)$ has the same value in every infinite dimensional structure elementary equivalent to a given s.m. one and

(ii)rk $\varphi(M)$ does not depend on the choice of parameters $A$ over which $\varphi(M)$ is definable.

Proof Suppose rk $\varphi(M)=r$ and $\left\{s_{1}, \ldots, s_{r}\right\}$ is a basis of $\left\langle s_{1}, \ldots s_{n}\right\rangle \in \varphi(M)$ of maximal dimension over $A$. If $A \cup A^{\prime} \subseteq M^{\prime}, M^{\prime}$ of infinite dimension and $M^{\prime} \equiv \equiv_{A \cup A^{\prime}} M$ then choose $\left\{s_{1}^{\prime}, \ldots, s_{r}^{\prime}\right\} \subseteq M^{\prime}$ independent over $A \cup A^{\prime}$. Since $s_{i}$, for all $i \leq n$, are algebraic over $\left\{s_{1}, \ldots, s_{r}\right\}$ the type

$$
p_{s_{1}, \ldots, s_{r}}=\operatorname{tp}\left(\left\langle s_{1}, \ldots, s_{n}\right\rangle /\left\{s_{1}, \ldots, s_{r}\right\} \cup A\right)
$$

is algebraic and principal. Since $\operatorname{tp}\left(\left\langle s_{1}, \ldots, s_{r}\right\rangle / A\right)=\operatorname{tp}\left(\left\langle s_{1}^{\prime}, \ldots, s_{r}^{\prime}\right\rangle / A\right)$, the type $p_{s_{1}^{\prime}, \ldots, s_{r}^{\prime}}$ obtained by substituting $\left\{s_{1}^{\prime}, \ldots, s_{r}^{\prime}\right\}$ for $\left\{s_{1}, \ldots, s_{r}\right\}$ in all the formulas of $p_{s_{1}, \ldots, s_{r}}$, is algebraic and principal too. Hence it has a realisation $\left\langle s_{1}^{\prime}, \ldots, s_{n}^{\prime}\right\rangle$ which the type says is a point in $\varphi\left(M^{\prime}\right)$ of dimension $r$ over $A \cup A^{\prime}$. This proves that rk $\varphi(M) \leq$ rk $\varphi\left(M^{\prime}\right)$. By symmetry we have rk $\varphi(M) \leq \operatorname{rk} \varphi\left(M^{\prime}\right)$, and the rank remains the same when we extend the set of parameters from $A$ to $A \cup A^{\prime}$. Then also the rank has to be the same as defined over $A^{\prime}$. 
Definition For an arbitrary s.m. structure $M, \operatorname{rk} \varphi(M)$ is defined as the rank in a saturated elementary extensions of $M$.

Lemma 4.2.2 (Basic Rank Properties) For any s.m.structure $M$

(i) $\mathrm{rk} M^{n}=n$;

(ii) rk $S=0$ iff $S$ is finite

(iii) $\operatorname{rk}\left(S_{1} \cup S_{2}\right)=\max \left\{\operatorname{rk} S_{1}\right.$, rk $\left.S_{2}\right\}$

(iv) For projection pr: $M^{n} \rightarrow M^{k}$

$$
\operatorname{rk} S \leq \operatorname{rk} \operatorname{pr}(S)+\max _{t \in \operatorname{pr}(S)} \operatorname{rkpr}^{-1}(t) .
$$

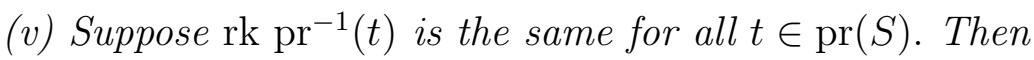

$$
\text { rk } S=\operatorname{rk} \operatorname{pr}(S)+\mathrm{rk} \mathrm{pr}^{-1}(t) .
$$

Proof (i)-(iii) are immediate from definition.

(iv) Let $\left\langle s_{1}, \ldots s_{n}\right\rangle \in S$ be of maximal dimension in $S$. Then

$$
\begin{gathered}
\operatorname{rk} S=\operatorname{dim}\left(\left\{s_{1}, \ldots s_{n}\right\} / A\right)=\operatorname{dim}\left(\left\{s_{1}, \ldots s_{n}\right\} /\left\{s_{1}, \ldots s_{k}\right\} \cup A\right)+ \\
\operatorname{dim}\left(\left\{s_{1}, \ldots s_{k}\right\} / A\right) \leq \operatorname{rk~pr}^{-1}\left(\left\langle s_{1}, \ldots s_{k}\right\rangle\right)+\operatorname{rk} \operatorname{pr} S
\end{gathered}
$$

(v) If one chooses first a generic $\left\langle s_{1}, \ldots s_{k}\right\rangle \in \operatorname{pr} S$ and then extends it to $\left\langle s_{1}, \ldots s_{n}\right\rangle \in S$ of maximal possible dimension over $\left\{s_{1}, \ldots s_{k}\right\} \cup A$, then

$$
\begin{aligned}
\operatorname{dim}\left(\left\{s_{1}, \ldots s_{n}\right\} /\left\{s_{1}, \ldots s_{k}\right\} \cup A\right) & =\operatorname{rkpr}^{-1}\left(\left\langle s_{1}, \ldots s_{k}\right\rangle\right), \\
\operatorname{dim}\left(\left\{s_{1}, \ldots s_{k}\right\} / A\right) & =\operatorname{rkpr} S
\end{aligned}
$$

and thus

$$
\operatorname{rk} S \geq \operatorname{rkpr} S+\operatorname{rkpr}^{-1}\left(\left\langle s_{1}, \ldots s_{k}\right\rangle\right)
$$


Lemma 4.2.3 For any definable $S \subseteq M^{n}$ there is an upper bound for $m \in \mathbb{N}$ such that $S$ can be partitioned into $k$ disjoint subsets

$$
S=S_{1} \cup \cdots \cup S_{m}
$$

each of rank equal to rk $S$.

Proof We use induction on $n$. For $n=1$ the statement follows by definition. For arbitrary $n$ let rk $S=k$. This means there is a point $\left\langle s_{1}, \ldots s_{n}\right\rangle \in S$ of dimension $k$ and thus some $\left\{s_{i_{1}}, \ldots s_{i_{k}}\right\}$ are independent. Let us assume $\left\langle i_{1}, \ldots i_{k}\right\rangle=\langle 1, \ldots k\rangle$.

Then $s_{j} \in \operatorname{acl}\left\{s_{1}, \ldots s_{k}\right\}$ for all $j=1, \ldots, n$, thus for some natural number $l=l_{i_{1}, \ldots i_{k}}$

$$
M \models \exists^{=l}\left\langle x_{1}, \ldots x_{n}\right\rangle \in S:\left\langle x_{1}, \ldots x_{k}\right\rangle=\left\langle s_{1}, \ldots s_{k}\right\rangle .
$$

Denote the formula

$$
\psi\left(y_{1}, \ldots y_{k}\right):=\exists^{=l}\left\langle x_{1}, \ldots x_{n}\right\rangle \in S:\left\langle x_{1}, \ldots x_{k}\right\rangle=\left\langle y_{1}, \ldots y_{k}\right\rangle
$$

and notice that $\psi(M) \subseteq M^{k}$ is of rank $k$. Let

$$
S^{i_{1}, \ldots i_{k}}=\left\{\left\langle s_{1}, \ldots s_{n}\right\rangle \in S: \psi\left(s_{1}, \ldots s_{k}\right)\right\}
$$

Calculating by rank properties above we get $\operatorname{rk} S^{i_{1}, \ldots i_{k}}=\operatorname{rk} \psi(M)=\operatorname{rk} S$. Suppose

$$
S^{i_{1}, \ldots i_{k}}=S_{1} \cup \cdots \cup S_{p}
$$

is a partition and all the summands are $A^{\prime}$-definable of rank $k$, some $A^{\prime}$. Then necessarily for any $j \leq p$ there is $\left\langle s_{j, 1}, \ldots s_{j, n}\right\rangle \in S_{j}$ with the first $k$ coordinates independent over $A^{\prime}$. By indiscernibility we can choose $\left\langle s_{j, 1}, \ldots s_{j, n}\right\rangle \in$ $S_{j}^{0}$ so that

$$
\left\langle s_{j, 1}, \ldots s_{j, k}\right\rangle=\left\langle s_{1}, \ldots s_{k}\right\rangle
$$

for all $j$. It follows immediately that $p \leq l$.

Taking into account all possibilities for $\left\langle i_{1}, \ldots i_{k}\right\rangle$ we get

$$
m \leq \sum_{\left\{i_{1}, \ldots i_{k}\right\}} l_{i_{1}, \ldots i_{k}}
$$


Remark The definition of rank makes sense for any $\lambda$-minimal structure of infinite dimension.

Part (ii) of Lemma 4.2.1 holds for every $\lambda$-minimal $\aleph_{0}$-homogeneous structure with the same proof.

Correspondingly for such structures the statement of 4.2 .3 takes form

Exercise 4.2 Given $S$ of rank $k$, any family $\left\{S_{j}: j \in J\right\}$ of pairwise disjoint definable subsets of rank $k$ of $S$ is of cardinality less than $\lambda$.

Example The rank notion makes sense in $\mathbb{C}^{e}$ and the statement of the Exercise is in close connection with the fact that any analytic subset has at most countably many irreducible components.

Definition The exact upper bound for equirank partition of $S$ is called the (Morley) degree of $S$.

A definable set of degree one is said to be irreducible.

Definition For a type $p(\bar{x})$ definable over $A$ Morley rank of type is defined as

$$
\operatorname{rk}(p)=\min \{\operatorname{rk} \varphi(\bar{x}): \varphi \in p\} .
$$

For a point $\bar{s} \in M^{n}$ and a subset $A \subseteq M$ the Morley rank of the point over $A$ is defined as

$$
\operatorname{rk}(\bar{s} / A)=\operatorname{rk}(\operatorname{tp}(\bar{s} / A)) .
$$

Given an irreducible subset $S$ defined over $A$, a point $\bar{s} \in S$ is said to be generic over $A$ if

$$
\operatorname{rk}(\bar{s} / A)=\operatorname{rk} S .
$$

Exercise $4.3 \mathrm{rk}\left(\left\langle s_{1}, \ldots, s_{n}\right\rangle / A\right)=\operatorname{dim}\left(\left\{s_{1}, \ldots, s_{n}\right\} / A\right)$.

Lemma 4.2.4 For $S$ irreducible defined over $A$ there is a unique complete type $p$ over A containing $S$ of rank rk $S$. More exactly,

$$
p=\operatorname{tp}(s / A)
$$

for s generic in $S$. In particular, any two generic points have the same type over $A$. 
Proof By irreducibility

$$
p=\{\varphi(\bar{x}) \text { over } A: \operatorname{rk}(\varphi(M) \cap S)=\operatorname{rk} S\}
$$

is a type. The rest follows from definitions.

\section{Lemma 4.2.5 (The addition formula for tuples)}

$$
\operatorname{rk}(\bar{b} \bar{c} / A)=\operatorname{rk}(\bar{b} / A \bar{c})+\operatorname{rk}(\bar{c} / A)
$$

(Here $A \bar{c}=A \cup|\bar{c}|,|\bar{c}|$ is the set of the coordinates of $\bar{c}$.)

Proof This is just another form of the addition formula for dimensions 3.1.3 $\square$

Definition Two points $\bar{b} \in M^{k}$ and $\bar{c} \in M^{n}$ are said to be independent over $A$ if

$$
\operatorname{rk}(\bar{b} / A \bar{c})=\operatorname{rk}(\bar{b} / A) .
$$

Lemma 4.2.6 The independence relation is symmetric

Proof rk $(\bar{b} \bar{c} / A)=\operatorname{rk}(\bar{b} / A \bar{c})+\operatorname{rk}(\bar{c} / A)=\operatorname{rk}(\bar{c} / A \bar{b})+\operatorname{rk}(\bar{b} / A)$ by the addition formula. Then $\mathrm{rk}(\bar{b} / A \bar{c})=\operatorname{rk}(\bar{b} / A)$ implies $\operatorname{rk}(\bar{c} / A \bar{b})+\operatorname{rk}(\bar{c} / A)$.

Lemma 4.2.7 (Definability of Morley Rank) For any formula $\varphi(\bar{x}, \bar{y})$ with length $(\bar{x})=k$, length $(\bar{y})=n$, and any $m$ the set

$$
\left\{\bar{a} \in M^{k}: \operatorname{rk} \varphi(\bar{a}, M) \geq m\right\}
$$

is definable.

Proof By induction on $n$. For $n=1$ rk $\varphi(a, M) \geq 0$ iff $\varphi(a, M) \neq \emptyset$, and $\operatorname{rk} \varphi(a, M) \geq 1$ iff $\varphi(a, M)$ is infinite iff $\operatorname{card} \varphi(a, M) \geq n_{\varphi}$ by strong definability of finiteness (not f.c.p.).

For arbitrary $n$

rk $\varphi\left(a, y_{1}, \ldots, y_{n}\right) \geq m$ iff $\left\{b \in M:\right.$ rk $\left.\varphi\left(a, b, y_{2}, \ldots, y_{n}\right) \geq m-1\right\}$ is infinite or $\left\{b \in M: \operatorname{rk} \varphi\left(a, b, y_{2}, \ldots, y_{n}\right) \geq m\right\} \neq \emptyset$

by the addition formula for ranks. The both conditions on the right hand side are definable by induction hypothesis. 


\subsection{Sets definable in $M$.}

We shall consider Morley rank for sets definable in strongly minimal $M$. Recall that any such set is of the form $U=S / E$, where $S \subseteq M^{n}$ is a definable subset and $E \subseteq S^{2} \subseteq M^{2 n}$ is a definable subset which is an equivalence relation. We consider only $U$ such that $E$ is equirank, i.e. rk $E(s, M)$ is of the same value for all $s \in S$.

Definition For $U=S / E$ definable in $M$ with $E$ an equivalence relation with equirank classes

$$
\operatorname{rk} U=\operatorname{rk} S-\operatorname{rk} E(s, M) \text { for } s \in S .
$$

Lemma 4.3.1 The definition is invariant under definable bijections, i.e. if there is a bijection

$$
f: S_{1} / E_{1} \rightarrow S_{2} / E_{2}
$$

and $f$ is a definable function, then $\mathrm{rk} S_{1} / E_{1}=\mathrm{rk} S_{2} / E_{2}$.

Proof By definition $f=F / E$, where $F \subseteq S_{1} \times S_{2}, \quad E=E_{1} \times E_{2}$ and the following hold

for any $s_{1}, s_{1}^{\prime} \in S_{1}, s_{2}, s_{2}^{\prime} \in S_{2}$

$$
\begin{gathered}
F\left(s_{1}, s_{2}\right) \& F\left(s_{1}^{\prime}, s_{2}^{\prime}\right) \Rightarrow\left(E_{1}\left(s_{1}, s_{1}^{\prime}\right) \leftrightarrow E_{2}\left(s_{2}, s_{2}^{\prime}\right)\right), \\
\operatorname{pr}_{S_{1}} F=S_{1} \text { and } \operatorname{pr}_{S_{2}} F=S_{2} .
\end{gathered}
$$

From the addition formula, projecting on $S_{1}$, we get

rk $F=\operatorname{rk} S_{1}+\mathrm{rk} E_{2}\left(s_{2}, M\right)$

and projecting on $S_{2}$

$\operatorname{rk} F=\operatorname{rk} S_{2}+\mathrm{rk} E_{1}\left(s_{1}, M\right)$.

It follows

$\operatorname{rk} S_{1}-\operatorname{rk} E_{1}\left(s_{1}, M\right)=\operatorname{rk} S_{2}-\operatorname{rk} E_{2}\left(s_{2}, M\right)$. 
Proposition 4.1 Basic Rank Properties (i)-(v) as well as Lemma 4.2.3 and definability of rank hold for definable sets.

Proof Follow the proofs of the statements mentioned.

Notation For definable $S_{1}, S_{2} \subseteq M^{n}$

$$
S_{1} \sqsubset S_{2} \Leftrightarrow \operatorname{rk}\left(S_{1} \backslash S_{2}\right)<\operatorname{rk} S_{1}
$$

and

$$
S_{1} \sqsubset \sqsupset S_{2} \Leftrightarrow S_{1} \sqsubset S_{2} \& S_{2} \sqsubset S_{1} .
$$

We also say in words that $S_{1}$ is almost a subset of $S_{2}$ or $S_{1}$ almost coincides with $S_{2}$, correspondingly.

Proposition 4.2 (Finite Equivalence Relation Theorem) For any $A$ definable set $S$ of rank $k$ there is an A-definable subset $S^{0} \subseteq S$ and an equivalence relation $E$ on $S^{0}$ such that $S^{0} \sqsubset \sqsupset S, S^{0} / E$ is finite and each equivalence class is of rank $k$ and irreducible.

Proof Let Morley degree of $S$ be $m$ and

$$
S=\bigcup_{i \leq m} \Psi_{i}\left(b_{i}, M\right)
$$

be the partition of $S$ into irreducible subsets of rank $k$ with $\Psi_{i}(z, x) A$ definable formulas and $b_{i}$ ranging in $M^{n}$ for some $n$. Our aim is to define an equivalence relation with $m$ equirank classes such that each class almost coincides with one of $\Psi_{i}\left(b_{i}, M\right)$.

Claim 1. We may choose $\Psi_{i}=\Psi$ independently on $i$. To get this consider formula $\Psi$ of parameters $b_{1}, \ldots, b_{m}$ and $c_{1}, \ldots c_{m}$, where $c_{1}, \ldots c_{m}$ are some distinct elements in $M$ :

$$
\Psi(y, x) \equiv \bigwedge_{i \leq m}\left(y=c_{i} \rightarrow \Psi\left(b_{i}, x\right)\right) .
$$

Evidently, $\Psi\left(c_{i}, x\right) \equiv \Psi_{i}\left(b_{i}, x\right)$, which proves the claim. 
Put

$$
\begin{gathered}
P=\left\{b \in M^{n}: \Psi(b, M) \subseteq S \& \operatorname{rk} \Psi(b, M)=k \quad \&\right. \\
\left.\& \forall c \in M^{n}(\operatorname{rk}(\Psi(b, M) \cap \Psi(c, M))=k \rightarrow(\Psi(b, M) \sqsubset \Psi(c, M)))\right\} .
\end{gathered}
$$

This set is $A$-definable, $b_{i} \in P$ for all $i \leq m$, and for any $b \in P, \Psi(b, M)$ is an irreducible subset of $S$ of rank $k$.

Define an equivalence relation on $P$

$$
F\left(b, b^{\prime}\right) \quad \text { iff } \quad \Psi(b, M) \sqsubset \sqsupset \Psi\left(b^{\prime}, M\right) .
$$

There are exactly $m$ equivalence classes of $F$ with $b_{1}, \ldots, b_{m}$ representatives of the classes.

Claim 2. Let $b \in P, s \in \Psi(b, M)$ and $\operatorname{rk}(s / A b)=k$. Then

$$
F(b, M) \sqsubset \Psi(M, s) .
$$

Proof of Claim. Let $b^{\prime} \in F(b, M)$ be of maximal rank, i.e. rk $\left(b^{\prime} / A b s\right)=$ rk $F(b, M)$. Then rk $\left(b^{\prime} / A b s\right)=\mathrm{rk}\left(b^{\prime} / A b\right)$, which means $b^{\prime}$ and $s$ are independent over $A b$. Then rk $\left(s / A b b^{\prime}\right)=\operatorname{rk}(s / A b)=k$. Since $\Psi(b, M) \sqsubset \sqsupset \Psi\left(b^{\prime}, M\right)$ it follows $s \in \Psi\left(b^{\prime}, M\right)$. In other words $\Psi\left(b^{\prime}, s\right)$ holds for all such $b^{\prime}$ and hence $F(b, M) \sqsubset \Psi(M, s)$.

Claim 3. For any $s \in S$ with $\operatorname{rk}\left(s / A b_{1}, \ldots, b_{m}\right)=k$ there is exactly one class $F(b, M)$ such that $F(b, M) \sqsubset \Psi(M, s)$.

Indeed, suppose $F(b, M) \sqsubset \Psi(M, s)$ for $b=b_{1}$ and $b=b_{2}$. Choose $\left\langle b_{1}^{\prime}, b_{2}^{\prime}\right\rangle \in$ $F\left(b_{1}, M\right) \times F\left(b_{2}, M\right)$ of maximal rank over $A b_{1} b_{2} s$. Then rk $\left(b_{1}^{\prime} b_{2}^{\prime} / A b_{1} b_{2} s\right)=$ rk $F\left(b_{1}, M\right)+$ rk $F\left(b_{2}, M\right)=$ rk $\left(b_{1}^{\prime} b_{2}^{\prime} / A b_{1} b_{2}\right)$. It follows rk $\left(s / A b_{1} b_{2} b_{1}^{\prime} b_{2}^{\prime}\right)=$ rk $\left(s / A b_{1} b_{2}\right)$, and $\Psi\left(b_{1}^{\prime}, s\right) \& \Psi\left(b_{2}^{\prime}, s\right)$ holds. Hence rk $\left(\Psi\left(b_{1}^{\prime}, M\right) \& \Psi\left(b_{1}^{\prime}, M\right)\right)=$ $k$, contradicting $\neg F\left(b_{1}^{\prime}, b_{2}^{\prime}\right)$. Claim proved.

Define

$$
S^{0}=\{s \in S: \text { there is exactly one class } F(b, M) \sqsubset \Psi(M, s)\}
$$

and $E$ on $S^{0}$ to be

$$
E\left(s_{1}, s_{2}\right) \text { iff } \exists b \in P \quad F(b, M) \sqsubset \Psi\left(M, s_{1}\right) \cap \Psi\left(M, s_{2}\right) .
$$

By definition and claim 3 there is a one-to-one correspondence between $E$ classes and $F$-classes. By Claim $2 \Psi\left(b_{i}, M\right) \sqsubset E\left(s_{i}, M\right)$ for any generic $s_{i} \in \Psi\left(b_{i}, M\right)$ for all $i \leq m$. Thus $\Psi\left(b_{i}, M\right) \sqsubset \sqsupset E\left(s_{i}, M\right)$. 
Corollary 4.3 For A-definable $S$ as above there is a finite A-definable set $S_{0}$ such that any irreducible equirank subset of $S$ almost coinsides with an $A b$-definable subset for $b \in S_{0}$.

Exercise Generalise results of this section to $\lambda$-minimal $\aleph_{0}$-homogeneous structures with $\lambda$-smallness weakly definable. 


\section{Macro-geometries and the Trichotomy The- orem}

We explore further the geometries of minimal structures. We show in this section that in cases when the geometry of a minimal structure becomes too complicated for analysis, another type of geometry play an important role. We call the new geometries macro-geometry as opposed to micro-geometries introduced in section 3 .

\subsection{Macro- and micro-geometries on a s.m. structure.}

Example 1. Macro-geometry of an algebraically closed field. Let $F$ be a field and $\mathbf{A}^{2}(F)$ an affine plane over the field, i.e. $\mathbf{A}^{2}(F)=F \times F$ as a set of points. Let also $L^{2}(F)$ be the set of straight lines on $\mathbf{A}^{2}(F)$ that can be identified as the set of triples $(a, b, c) \in F^{3},(a, b) \neq(0,0)$, modulo the equivalence relation $E$ :

$$
(a, b, c) E\left(a^{\prime}, b, c\right) \quad \text { iff } \quad \exists \lambda \neq 0 \quad(a, b, c)=\lambda\left(a^{\prime}, b^{\prime}, c^{\prime}\right)
$$

which define a straight line by equation

$$
a x+b y+c=0 .
$$

If we also add a triple $(0,0,1)$ to $L^{2}(F)$ we get the classical projective plane over $F$ denoted $\mathbf{P}^{2}(F)$.

$\left(\mathbf{A}^{2}, L^{2}\right)$ can be considered as a two-sorted structure with the incidence relation $I \subseteq \mathbf{A}^{2} \times L^{2}$ defined by

$$
(x, y) I(a, b, c) \text { iff } a x+b y=c .
$$

Through any two points there is a unique line. Any two lines intersect in at most one point and for almost any pair of lines the intersection is non-empty.

By the Main Theorem of Projective Geometry the field is definable in the structure $\left(\mathbf{A}^{2}, L^{2}, I\right)$, in other words the macro-geometry bears all the information on $F$. 
Example 2. Macro-geometry of a $k$-vector space. Let $k$ be a field and $V$ an infinite dimensional vector space over $k$. Consider $V^{2}$ as a set of points and, given $\alpha \in k$, define a line in $V^{2}$ through a point $(a, b)$ with a slope $\alpha$ to be

$$
\left\{(x, y) \in V^{2}: \alpha(x-a)=y-b\right\} .
$$

We have as many lines through $(a, b)$ as there are elements in $k^{*}$, bounded independently on the cardinality of $V$.

If $k$ is a finite field the set of lines is definable in $V$ and indeed we can define again a two-sorted structure of 'points and lines' with an incidence relation $I$.

\section{Example 3. Macro-geometry of a trivial structure.}

Let $M$ be a set. Consider $M^{2}$ as a set of points and define a left line through $\langle a, b\rangle$ to be

$$
\left\{\langle x, y\rangle \in M^{2}: x=a\right\} .
$$

Correspondingly define the right line.

This geometry of points and lines is very simple. Lines almost never meet, and through almost no pair of points there is a common line.

Definition An [abstract] projective geometry is a set of 'points' and 'lines' satisfying:

(i) through any two points there is a line;

(ii) there are at least three points on every line;

(iii) two distinct lines intersect in at most one point;

(iv) for any distinct points $a, b, c, d$ : if lines $(a, b)$ and $(c, d)$ intersect then lines $(a, c)$ and $(b, d)$ do.

Any 3 points $a, b, c$ of a projective geometry which do not lie on a common line generate a projective plane as the set of points

$$
S(a, b, c)=\bigcup\{(a, z): z \in(b, c)\} .
$$

By (iv) the plane generated by any non-collinear $a^{\prime}, b^{\prime}, c^{\prime} \in S(a, b, c)$ coincides with $S(a, b, c)$. The $n$-subspaces of a projective geometry are defined by induction as

$$
S\left(a_{1}, \ldots, a_{n+1}\right)=\bigcup\left\{\left(a_{n+1}, z\right): z \in\left(a_{1}, \ldots, a_{n}\right)\right\}
$$

for $a_{1}, \ldots, a_{n+1}$ not in a $(n-1)$-subspace. Again by axiom (iv) the definition is invariant on the choice of the points in the subspace. 
Theorem 5.1 Any projective geometry of dimension greater than two (generated by no less than 4 points) is isomorphic to a projective geometry over a division ring.

Proof See [Se].

Motivated by the definitions above A.Lachlan has introduced the notion of a pseudoplane. We specialise the notion for our purposes

Definition A rank-two pseudo-plane is a two-sorted structure $(P, L)$ of 'points and lines' definable in a strongly minimal structure $M$ with an incidence relation $I \subseteq P \times L$ such that

$P$ is irreducible, rk $P=2$;

rk $L \geq 2$;

rk $I l=1$ for all $l \in L$;

if $l_{1}, l_{2} \in L, \quad l_{1} \neq l_{2}$ then $I l_{1} \cap I l_{2}$ is finite or empty.

(Here $I l=\{p \in P: \vDash p I l\}$.)

Proposition 5.1 Suppose $M$ is a strongly minimal structure and there are $a_{1}, a_{2}, b_{1}, b_{2}, c \in M$ every four of which are independent, $c \in \operatorname{acl}\left(a_{1}, a_{2}, b_{1}, b_{2}\right)$ and

$$
\operatorname{acl}\left(a_{1}, a_{2}, c\right) \cap \operatorname{acl}\left(b_{1}, b_{2}, c\right)=\operatorname{acl}(c) .
$$

Then a rank-two pseudoplane is definable in $M$.

Remark The condition in the Proposition can be stated more geometrically in the localisation $M_{c}$ of the micro-geometry of $M$ : the lines $\left(a_{1}, a_{2}\right)$ and $\left(b_{1}, b_{2}\right)$ of the micro-geometry lie on the same plane $\left(a_{1}, a_{2}, b_{1}, b_{2}\right)$ but do not meet. In other words the micro-geometry on $\left(a_{1}, a_{2}, b_{1}, b_{2}\right)$ is not that of the projective plane.

Proof of the proposition. Put $P_{0}=M \times M, L_{0}=M \times M \times M$, and let $I_{0} \subseteq P_{0} \times L_{0}$ be an $\emptyset$-definable relation such that

$$
\left\langle b_{1}, b_{2}\right\rangle I_{0}\left\langle a_{1}, a_{2}, c\right\rangle
$$




$$
\left\langle x_{1}, x_{2}\right\rangle I_{0}\left\langle y_{1}, y_{2}, z\right\rangle \rightarrow z \in \operatorname{acl}\left(x_{1}, x_{2}, y_{1}, y_{2}\right) .
$$

A definable relation witnessing the dependence between $a_{1}, a_{2}, b_{1}, b_{2}, c$ has these properties.

Our first aim is to transform the incidence relation we have defined to an incidence relation with iinfinite lines meeting in at most finitely many points. Denote $l_{0}=\left\langle a_{1}, a_{2}, c\right\rangle$. Then $I_{0} l_{0}$ is an $l_{0}$-definable set of Morley rank 1 .By the Finite Equivalence Relation Theorem using $l_{0}$ one can define an equivalence relation $E_{l_{0}}$ on $I_{0} l_{0}$ with finitely many classes, and, say $m$ of them of rank 1 irreducible.

Denote

$$
I_{1}=\left\{\langle p, l\rangle \in I_{0}: E_{l} \text { is an equivalence relation on } I_{0} l\right. \text { with exactly }
$$

$m$ infinite classes and $p$ is in one of the infinite classes $\}$.

Denote a binary relation $E$ on $I_{1}$ :

$$
\langle p, l\rangle E\left\langle p^{\prime}, l^{\prime}\right\rangle \text { iff } l=l^{\prime} \& p E_{l} p^{\prime} .
$$

Define

$$
L_{1}=I_{1} / E
$$

and for $k \in L_{1}, p \in P_{0}$ write $p I_{2} k$ iff $k=\langle p, l\rangle$ for some $l \in L_{0}$.

By definitions there is a canonical mapping

$$
\alpha: L_{1} \rightarrow L_{0}
$$

corresponding to the projection $I_{1} \rightarrow L_{0}$, which is exactly $m$-to-one mapping. Also,

$$
\text { for all } k \in L_{1} \quad \mathrm{rk}\left(I_{2} k\right)=1 \text {. }
$$

By definitions, for $k_{0}$ corresponding to $l_{0}$ via $\alpha, I_{2} k_{0}$ is irreducible.

Define

$$
L_{2}=\left\{k \in L_{1}: \forall k^{\prime} \in L_{1} \quad \mathrm{rk}\left(I_{2} k \cap I_{2} k^{\prime}\right)=1 \rightarrow I_{2} k \sqsubset I_{2} k^{\prime}\right\} .
$$

It follows from the above remark that $k_{0} \in L_{2}$ and, for all $k \in L_{2}, \operatorname{rk}\left(I_{2} k\right)=$ 1 .

Any two lines represented in $L_{2}$ either almost coincide or meet in at most finitely many points. 
Define an equivalence relation on $L_{2}$

$$
k F k^{\prime} \text { iff } I_{2} k \sqsubset \sqsupset I_{2} k^{\prime} .
$$

We are now in the situation of Claim 2 of the proof of the Finite Equivalence Relation Theorem. It follows that

$$
k F \sqsubset p I_{2}
$$

whenever $p$ is generic in $I_{2} k$ over $k$ and $p$ is generic in $P_{0}$.

Define

$$
L_{3}=L_{2} / F
$$

and, for $\bar{l} \in L_{3}, p \in P_{0}$,

$$
p I_{3} \bar{l} \text { iff } \bar{l} \sqsubset p I_{2} .
$$

From the above proved $p_{0} I_{3} \bar{l}_{0}$ holds, where $\bar{l}_{0}$ is obtained throughout the construction from $l_{0}, p_{0}$. Also, by the construction $\bar{l}_{0} \in \operatorname{acl}\left(l_{0}\right)$.

Since $p_{0} \in I_{3} \bar{l}_{0}$ it follows $\mathrm{rk}\left(I_{3} \bar{l}_{0}\right) \geq 1$. On the other hand, if $p \in I_{3} \bar{l}_{0}$ is of maximal rank over $\bar{l}_{0}$ and $k \in l_{0} F$ is of maximal rank over $p, \bar{l}_{0}$ then by definition $p I_{2} l$ holds and $k$ and $p$ are independent over $\bar{l}_{0}$. It follows rk $\left(p / \bar{l}_{0}\right)=$ rk $\left(p / \bar{l}_{0}, k\right) \leq 1$. Thus

$$
\operatorname{rk}\left(I_{3} \bar{l}_{0}\right)=1
$$

Let

$$
\begin{gathered}
L=\left\{\bar{l} \in L_{3}: \operatorname{rk}\left(I_{3} \bar{l}_{0}\right)=1\right\}, \quad P=\left\{p \in P_{0}: \exists \bar{l} \in L p I_{3} \bar{l}\right\}, \\
I=I_{3} \cap(P \times L) .
\end{gathered}
$$

Now we need to show that for distinct $\bar{l}_{1}, \bar{l}_{2}$ from $L \quad I \bar{l}_{1} \cap I \bar{l}_{2}$ is finite. So, suppose $p$ is a point in the intersection. Choose $\left\langle k_{1}, k_{2}\right\rangle \in l_{1} F \times l_{2} F$ of maximal rank over $p, \bar{l}_{1}, \bar{l}_{2}$. Then $p \in I_{2} k_{1} \cap I_{2} k_{2}$ and $p$ is independent from $k_{1}, k_{2}$ over $\bar{l}_{1}, \bar{l}_{2}$. Then

$$
\operatorname{rk}\left(p / \bar{l}_{1}, \bar{l}_{2}\right)=\operatorname{rk}\left(p / \bar{l}_{1}, \bar{l}_{2}, k_{1}, k_{2}\right)<1
$$

since $\neg k_{1} F k_{2}$.

To finish the proof we need to show that rk $(L) \geq 2$ which would follow from rk $\left(\bar{l}_{0} / \emptyset\right) \geq 2$.

Suppose towards the contradiction $\mathrm{rk}\left(\bar{l}_{0} / \emptyset\right) \leq 1$. Then, since $\mathrm{rk}\left(p_{0} / \bar{l}_{0}\right)=$ $1<\operatorname{rk}\left(p_{0} / \emptyset\right)$, we have $\operatorname{rk}\left(\bar{l}_{0} / p_{0}\right)<\operatorname{rk}\left(\bar{l}_{0} / \emptyset\right)$, i.e. $\bar{l}_{0} \in \operatorname{acl}\left(p_{0}\right)=\operatorname{acl}\left(b_{1}, b_{2}\right)$. Then, from the assumptions of the proposition $c \notin \operatorname{acl}\left(\bar{l}_{0}\right)$. 
On the other hand $\bar{l}_{0} \in \operatorname{acl}\left(l_{0}\right)=\operatorname{acl}\left(a_{1}, a_{2}, c\right)$. It follows $b_{1} \notin \operatorname{acl}\left(l_{0}\right), b_{2} \notin$ $\operatorname{acl}\left(l_{0}\right)$. Therefore there exists $c^{\prime} \in M$ such that

$$
\operatorname{tp}\left(c c^{\prime} / \bar{l}_{0}\right)=\operatorname{tp}\left(b_{1} b_{2} / \bar{l}_{0}\right)=\operatorname{tp}\left(p_{0} / \bar{l}_{0}\right) .
$$

Thus rk $\left(c c^{\prime} / \bar{l}_{0}\right)=1$ and so

$$
c^{\prime} \in \operatorname{acl}\left(\bar{l}_{0}, c\right) \subseteq \operatorname{acl}\left(a_{1}, a_{2}, c\right) \cap \operatorname{acl}\left(b_{1}, b_{2}, c\right) .
$$

Hence $\operatorname{acl}\left(c^{\prime}\right)=\operatorname{acl}(c)$, contradicting $\operatorname{acl}\left(b_{1}\right) \neq \operatorname{acl}\left(b_{2}\right)$.

Remark Note that $P$ and $L$ are 'coordinatised by $M$ in a very strong sense. That is $P$ is a subset of $M \times M$, by construction and $L$ is in finite to finite correspondence with $M \times M$. To see the latter note that from the very beginning of the construction we could replace $L_{0}$ by $L_{0}(c)=M \times M \times\{c\}$, and carry on with the same construction to obtain $L_{1}(c), L_{2}(c), L_{3}(c)$ and finally $L(c)$. The observation at the end of the proof that $c \notin \operatorname{acl}\left(\bar{l}_{0}\right)$ implies that almost all equivalence classes are represented in the smaller sets, that is rk $L(c)=2$ and $L(c)$ is in finite to finite correspondence with a rank 2 subset of $L$.

\subsection{The Trichotomy Theorem}

The condition detected in the Proposition 5.1 can be interpreted as an abstract notion of non-linearity of the geometry. The alternative to the condition is, as follows from the remark to the proposition, that the planes of the geometry of $M$ localised in a generic point are abstract projective or trivial. In this section we study the trichotomy in more details.

Theorem 5.2 (weak Trichotomy Theorem) For any strongly minimal $M$ either

(i) a rank-two pseudoplane is definable in $M$

or one of the following hold:

(ii) the geometry of $M$ is trivial, i.e. for any $X \subseteq \hat{M}, \operatorname{acl}(X)=(X)$ in $\hat{M}$;

(iii) the geometry of $M$ is locally projective, i.e. for generic $c \in M$ the geometry $\hat{M}_{c}$ is isomorphic to a projective geometry over a division ring. 
Proof Assume no pseudoplane is definable in $M$ and $c$ is a fixed generic element in $M$.

Claim 1. For any $x, y \in M$ and $Z \subseteq M$ finite

$$
x \in \operatorname{acl}(y, c, Z) \text { implies } \exists z \in \operatorname{acl}(c, Z): x \in \operatorname{acl}(y, z, c) \text {. }
$$

We may assume that $Z$ is independent over $c$ and proceed by induction on $\# Z$. For $\# Z=1$ there is nothing to prove.

Suppose $Z=\left\{z_{1}, z_{2}\right\} \cup Z^{\prime}, \quad x \in \operatorname{acl}(y, c, Z), y \notin \operatorname{acl}(c, Z)$. Then, by Proposition 5.1, in $M_{Z^{\prime}}$ either

(i) some quadruple from $x, y, z_{1}, z_{2}, c$ is dependent or

(ii) $\exists z \in M \backslash \operatorname{acl}_{Z^{\prime}}(c)$

$$
\operatorname{acl}_{Z^{\prime}}\left(z_{1}, z_{2}, c\right) \cup \operatorname{acl}_{Z^{\prime}}(x, y, c) \supseteq \operatorname{acl}_{Z^{\prime}}(z, c) .
$$

In case (i) only $x \in \operatorname{acl}_{Z^{\prime}}\left(y, z_{1}, z_{2}\right)$ is non-trivial. Which means in $M x \in$ $\operatorname{acl}\left(y, z_{1},\left\{z_{2} Z^{\prime}\right\}\right)$. Since $\#\left\{z_{2}, Z^{\prime}\right\}<\# Z$ by induction hypothesis there is $z \in \operatorname{acl}\left(z_{1}, z_{2}, Z^{\prime}\right): x \in \operatorname{acl}\left(y, z_{1}, z\right)$. If then $\operatorname{dim}\left(y, z_{1}, z, c\right)=3$, we have $x \in \operatorname{acl}\left(y, z_{1}, c\right)$ or $x \in \operatorname{acl}(y, z, c)$ and we get the desired. Otherwise, there is a point $z_{1}^{\prime} \in \operatorname{acl}\left(z_{1}, z, c\right) \backslash\left(\operatorname{acl}\left(z_{1}, z\right) \cup \operatorname{acl}\left(c, z_{1}\right) \cup \operatorname{acl}(c, z)\right)$. Assuming $x \notin$ $\operatorname{acl}(y, z)$ we have then that any four points of $\left\{x, y, z, z_{1}^{\prime}, c\right\}$ are independent. Again, using the Proposition and our assumption, there must exist $z^{\prime} \in$ $M \backslash \operatorname{acl}(c)$ such that

$$
\operatorname{acl}\left(z, z_{1}^{\prime}, c\right) \cap \operatorname{acl}(x, y, c) \supseteq \operatorname{acl}\left(z^{\prime}, c\right) .
$$

Clearly $z^{\prime} \in \operatorname{acl}(c, Z) \cap \operatorname{acl}(x, y, c)$, so $x \in \operatorname{acl}\left(y, z^{\prime}, c\right)$ and we are done.

In case (ii) $z \in \operatorname{acl}(c, Z)$ and $x \in \operatorname{acl}_{Z^{\prime}}(y, z, c)$, i.e. $x \in \operatorname{acl}\left(y, c, z, Z^{\prime}\right)$. By the induction hypothesis there is $z^{\prime} \in \operatorname{acl}\left(c, z, Z^{\prime}\right)$ such that $x \in \operatorname{acl}\left(y, z^{\prime}, c\right)$. Claim proved.

Claim 2. If $\operatorname{acl}(x, y, c)=\operatorname{acl}(x, c)=\operatorname{acl}(y, c)$ for some $x, y$ independent over $c$ then the geometry $\hat{M}$ is degenerate, i.e.

$$
\operatorname{acl}\left(x_{0}, \ldots, x_{n}\right)=\operatorname{acl}\left(x_{0}\right) \cup \cdots \cup \operatorname{acl}\left(x_{n}\right)
$$

for any $x_{0}, \ldots, x_{n} \in M$.

Indeed, under the assumption, $\operatorname{acl}\left(x_{0}, x_{1}, x_{2}\right)=\operatorname{acl}\left(x_{0}, x_{1}\right) \cup \operatorname{acl}\left(x_{0}, x_{2}\right)$ for any independent triple. We show first that the claim is true for $n=1$. 
Assume towards a contradiction $y \in \operatorname{acl}\left(x_{1}, x_{2}\right) \backslash\left(\operatorname{acl}\left(x_{1}\right) \cup \operatorname{acl}\left(x_{2}\right)\right)$. Choose $x_{0} \notin \operatorname{acl}\left(x_{1}, x_{2}\right)$. Then

$$
y \in \operatorname{acl}\left(x_{0}, x_{1}, x_{2}\right)=\operatorname{acl}\left(x_{0}, x_{1}\right) \cup \operatorname{acl}\left(x_{0}, x_{2}\right) .
$$

But if $y \in \operatorname{acl}\left(x_{0}, x_{i}\right)$ for $i=1$ or $i=2$ then $x_{0} \in \operatorname{acl}\left(y, x_{i}\right)=\operatorname{acl}\left(x_{1}, x_{2}\right)$, the contradiction.

Now we proceed by induction on $n$. Suppose $y \in \operatorname{acl}\left(x_{0}, \ldots, x_{n}\right)$. Then by Claim 1 there is $x \in \operatorname{acl}\left(x_{0}, \ldots, x_{n-1}\right)$ such that $y \in \operatorname{acl}\left(x_{n}, x, x_{0}\right)$. From what is proved already $y \in \operatorname{acl}\left(x, x_{0}\right) \cup \operatorname{acl}\left(x_{n}, x_{0}\right)$. Hence $y \in \operatorname{acl}\left(x_{0}, \ldots, x_{n-1}\right) \cup$ $\operatorname{acl}\left(x_{0}, x_{n}\right)=\operatorname{acl}\left(x_{0}, \ldots, x_{n-1}\right) \cup \operatorname{acl}\left(x_{n}\right) \cup \operatorname{acl}\left(x_{n}\right)=\operatorname{acl}\left(x_{0}\right) \cup \cdots \cup \operatorname{acl}\left(x_{n}\right)$.

This finishes the proof of the claim and of the Theorem.

\subsubsection{Geometries with finite closure property}

Definition A pregeometry (or a minimal structure) $M$ is said to have the finite closure property (sometimes also said locally finite) if for any finite set $X \subseteq M, \operatorname{cl}(X)$ is finite.

It is useful to note that if the theory of a structure $M$ is $\aleph_{0}$-categorical (or definable in such one) then, as a direct consequence of the Ryll-Nardzewski Theorem, $M$ has the finite closure property. In particular the following theorem fully characterises the geometries of strongly minimal structures definable in $\aleph_{0}$-categorical theories.

A very important consequence of the finite closure property is the possibility to introduce a stronger 'counting function' on definable sets.

Notation For a definable set $S$ in $M^{e q}$ and a subset $X \subseteq M$ denote

$$
S(X)=\operatorname{cl}(X) \cap S,
$$

where $\operatorname{cl}(X)$ is taken in $M^{e q}$. In particular, $M(X)=\operatorname{cl}(X) \cap M$.

Proposition 5.2 Assuming the finite closure property holds in a s.m. structure $M$, for every set $S$ in $M^{e q}$ over parameters $C$ there is a polynomial $p_{S} \in \mathbb{Q}[x]$ and a number $n_{S}$ such that for every finite $C \subseteq X \subseteq M$ : 
(i) letting $|M(X)|=x \geq n_{S}$, we have $|S(X)|=p_{S}(x)$;

(ii) $\mathrm{rk} S=\operatorname{deg} p_{S}$, the degree of the polynomial;

(iii) if $g(S)=T$ for some automorphism $g$ of $M$ then $p_{S}=p_{T}$ and $n_{S}=n_{T}$.

Proof We construct the polynomial for a given $S$.

W.l.o.g. we may assume that $S$ is an atom over $C$, that is defined by a principal type over $C$. It then has a form $S=U / E$ where $U \subseteq M^{n}$ is an atom over $C$ of rank $k \leq n$ and $E$ a $C$-definable equivalence relation on $U$.

Claim 1. For any finite $X \supseteq C$

$$
|U(X)|=m_{U}\left(x-u_{0}\right) \cdots\left(x-u_{k-1}\right)=p_{U}(x),
$$

where

$$
u_{0}=|M(C)|, \ldots, u_{k-1}=\left|M\left(C \cup\left\{d_{1}, \ldots, d_{k-1}\right\}\right)\right|, \ldots
$$

$d_{1}, \ldots, d_{k-1}$ any independent over $C$ elements of $M$, and given such $d_{1}, \ldots, d_{k-1}$,

$$
m_{U}=\left|\left\{\left\langle d_{k}, \ldots, d_{n}\right\rangle \in M^{n-k}:\left\langle d_{1}, \ldots, d_{k-1}, d_{k}, \ldots, d_{n}\right\rangle \in U\right\}\right|
$$

when we assume that enumeration of coordinates is such that, for $\left\langle a_{1}, \ldots, a_{n}\right\rangle \in$ $U, a_{1}, \ldots, a_{k}$ are independent over $C$.

Proof Indeed $U(X)$ consists of all possible $n$-tuples independent over $C$ in the first $k$ coordinates in $M(X)$ and the rest of the coordinates algebraic over them.

We also see from the description of $p_{U}$ that it is invariant under automorphisms and $n_{U}=0$.

As a corollary of the claim we have polynomials with the same properties for any definable subset of $M^{n}$.

Claim 2. Let $s \in S$ and $E_{s} \subseteq M^{n}$ be the $s$-definable $E$-equivalence class representing $s$. Then $p_{E_{s}}(x)=\left|E_{s}(X)\right|$ for any $x \geq n_{s}$, some $n_{s}$, and

$$
|S(X)|=\frac{p_{U}(x)}{p_{E_{s}}(X)}=p_{S}(x)
$$

for a polynomial $p_{S} \in \mathbb{Q}[x]$.

Proof Choose $n_{s}$ to be the cardinality of $M\left(C \cup\{s\} \cup\left\{d_{1}, \ldots, d_{l}\right\}\right)$ for a minimal $\left\{d_{1}, \ldots, d_{l}\right\} \subseteq M$ independent over $C \cup\{s\}$ such that $E_{s}(C \cup\{s\} \cup$ 
$\left.\left\{d_{1}, \ldots, d_{l}\right\}\right) \neq \emptyset$. Using automorphisms one sees that $E_{s}$ is an atom over $C \cup\{s\}$, thus the construction of Claim 1 applies and we get a polynomial

$$
p_{E_{s}}(x)=m_{s}\left(x-e_{0}\right) \cdots\left(x-e_{l-1}\right) \text {, }
$$

for some $e_{i}$ of the same description as $u_{j}$ in the definition of $p_{S}, l=\operatorname{rk} E_{s} \leq k$. More precisely $e_{0}=u_{r}, \ldots, e_{l-1}=u_{r+l-1}$ for some $r \leq k-l$. The claim and the proposition follow.

Notation We thus introduced a counting function $|S|=p_{S}$, the polynomial of a definable set $S$ and in particular $m(S)$ the leading coefficient of the polynomial $|S| . m_{S}$ is called also the (relative) multiplicity of $S$.

Theorem 5.3 The geometry of a minimal structure with the finite closure property is either trivial or isomorphic to an affine or a projective geometry over a finite field.

Idea of Proof. First notice that the structure is saturated and thus is strongly minimal. We first want to establish that the geometry of $M$ is locally projective or trivial. In order to do this, by the Trichotomy Theorem, we only need to prove that there is no two-rank pseudoplane in $M$. It is done by developing a combinatorial-geometric analysis of the pseudoplane $(P, I, L)$, assuming it exists.

The main tool of the analysis is the powerful rank notion $|S|$ introduced above for a structure with the finite closure property, or rather the multiplicity $m(S)$ in combination with the Morley rank rk $(S)$. See [Z] for the proof.

After one proves that $M$ is locally projective or trivial one can use a combinatorial result by Doyen and Hubaut which states that any finite locally projective geometry of dimension $\geq 4$ with equal number of points on all its lines is either affine or projective.

Corollary 5.1 Any geometry satisfying the finite closure property and the homogeneity assumption (any bijection between bases can be extended to an automorphism) is either trivial or isomorphic to an affine or a projective geometry over a finite field. 
Proof In an appropriate language such a geometry can be represented as a strongly minimal structure with finite closure property (see [Z]).

The weak Trichotomy Theorem generates the natural question: Does the definability of a rank-two pseudoplane in $M$ imply that the micro-geometry of $M$ is isomorphic to that of an algebraically closed field $F$. The answer is 'NO' in general (E.Hrushovski $[\mathrm{H}]$ ) and 'YES' if one assumes some extra topological-kind (Zariski) assumptions for $M$ (E.Hrushovski and B.Zilber [HZ]). Both results have been actively developed and applied in model theory and elsewhere in mathematics.

Problem Generalise results of this section to $\lambda$-minimal $\aleph_{0}$-homogeneous structures with $\lambda$-smallness weakly definable. 


\section{Categoricity in uncountable powers}

\subsection{Large models realising few types}

Stability theory started with the Ehrenfeucht-Mostowski Theorem which, rather characteristically for this field, is based on a fundamental theorem of infinite combinatorics

Ramsay's Theorem Let $A$ be an infinite set and

$$
A^{(n)}=\{X \subseteq A:|X|=n\}
$$

the set of all its n-element subsets. Suppose that

$$
A^{(n)}=C_{1} \dot{\cup} \ldots \dot{\cup} C_{k}
$$

is a partition into $k$ subsets. Then there are $a C_{i}$ and an infinite subset $B \subseteq A$ such that $B^{(n)} \subseteq C_{i}$.

Proof See [CK, Thm 3.3.7]

Definition An infinite subset $A \subseteq M$ with an order $<$ (not-necessarily definabe) of a structure $M$ is said to be an indiscernible sequence if for any

$a_{1}<\cdots<a_{n}$ and $a_{1}^{\prime}<\cdots<a_{n}^{\prime}$ from $A$

$$
\operatorname{tp}\left(a_{1}, \ldots, a_{n}\right)=\operatorname{tp}\left(a_{1}^{\prime}, \ldots, a_{n}^{\prime}\right) .
$$

Lemma 6.1.1 For any theory $T$ with infinite models there is a model $M$ with an infinite indiscernible sequence $A$.

Let $P(x),<$ be new names for a unary predicate and a binary relation

$T^{\prime}=T \cup\left\{{ }^{\prime}<^{\prime}\right.$ is a linear order on $\left.P\right\} \cup\left\{\forall a_{1}<\cdots<a_{n} \in P \forall a_{1}^{\prime}<\cdots<a_{n}^{\prime} \in P\right.$

$$
\left.\varphi\left(a_{1}, \ldots, a_{n}\right) \equiv \varphi\left(a_{1}^{\prime}, \ldots, a_{n}^{\prime}\right): \varphi \text { formulas of } L\right\} .
$$

Proof By Compactness Theorem it is enough to prove that for any finite set $\Delta$ of $L$-formulas $\varphi\left(x_{1}, \ldots, x_{n}\right)$ the theory

$$
T^{\Delta}=T \cup\left\{{ }^{\prime}<^{\prime} \text { is a linear order on } P\right\} \cup
$$




$$
\forall a_{1}<\cdots<a_{n} \in P \forall a_{1}^{\prime}<\cdots<a_{n}^{\prime} \in P \bigwedge_{\varphi \in \Delta} \varphi\left(a_{1}, \ldots, a_{n}\right) \equiv \varphi\left(a_{1}^{\prime}, \ldots, a_{n}^{\prime}\right) .
$$

Let $M$ be any infinite model of $T,<$ an arbitrary linear ordering of $M$ and $\left.M^{(} n\right)$ the set of all its $n$-subsets. We define an equivalence $E_{\Delta}$ on $\left.M^{(} n\right)$ letting

$$
\begin{gathered}
\left\{a_{1}<\cdots<a_{n}\right\} E_{\Delta}\left\{a_{1}^{\prime}<\cdots<a_{n}^{\prime}\right\} \text { iff } \\
\bigwedge_{\varphi \in \Delta} \varphi\left(a_{1}, \ldots, a_{n}\right) \equiv \varphi\left(a_{1}^{\prime}, \ldots, a_{n}^{\prime}\right) .
\end{gathered}
$$

There are obviously at most $2^{|\Delta|}$ classes of equivalence thus, by Ramsay's Theorem, there is an infinite subset $P \subseteq M$ such that any two $n$-tuples of elements of $P$ are equivalent. This $P$ satisfies $T^{\Delta}$.

Lemma 6.1.2 For any theory $T$ with infinite models, for any cardinal $\kappa$ there is a model $M$ with an indiscernible sequence $(A,<)$ order-isomorphic to $\kappa$.

\section{Proof Let}

$$
\left\{c_{\alpha}: \alpha \in \kappa\right\}
$$

be a set of new constant symbols and

$$
T^{\prime \prime}=T^{\prime} \cup\left\{P\left(c_{\alpha}\right): \alpha \in \kappa\right\} \cup\left\{c_{\alpha}<c_{\beta}: \alpha<\beta \in \kappa\right\}
$$

for $T^{\prime}$ as in the proof above. Then any finite subcollection of new formulas is consistent with $T^{\prime}$ by obvious reasons. Applying the Compactness Theorem again we get a model $M$ of $T^{\prime \prime}$ with the new constants distinguishing a subset of $P(M)$ indiscernible in the language $L$ and ordered as $\kappa$.

Theorem 6.1 (Ehrenfeucht-Mostowski) If a countable theory T has infinite models then for any infinite cardinal $\kappa$ there is a model $M$ of $T$ such that for any $B \subseteq M$ the number of complete 1-types over $B$ realised in $M$ is of cardinality at most card $B+\aleph_{0}$. 
Proof Let $\bar{T}$ be the theory with built-in Skolem functions extending $T$, and let $(A,<)$ be a well-ordered set of type $\kappa$. We again assume that $A \subseteq \mathcal{M}$ is a subset of a monster-model of $\bar{T}$ and let $\bar{M}(A)$ be a closure of $A$ under Skolem functions. Then $\bar{M}(X)$ is a model of $\bar{T}$ of cardinality $\kappa$ and $(A,<)$ an indiscernible sequence. Let $B \subseteq \bar{M}(A)$ and $C$ a subset of $A$ of cardinality $|B|+\aleph_{0}$ such that every $b \in B$ has a representation $b=t\left(c_{1}, \ldots, c_{k}\right)$ for some term $t$ and $c_{1}, \ldots, c_{k} \in C$.

We say that two $n$-tuples of $A, a_{1}<\cdots<a_{n}$ and $a_{1}^{\prime}<\cdots<a_{n}^{\prime}$ are equivalent over $C$ if for all $c \in C$

$$
a_{i}<c \text { iff } a_{i}^{\prime}<c .
$$

It follows that for every $L$-formula $\psi$, any $b_{1}=t_{1}\left(c_{1}, \ldots, c_{k}\right), \ldots, b_{m}=$ $t_{m}\left(c_{1}, \ldots, c_{k}\right) \in B$ and any two $n$-tuples $a_{1}<\cdots<a_{n}$ and $a_{1}^{\prime}<\cdots<a_{n}^{\prime}$ equivalent over $C$

$$
\begin{aligned}
& \vDash \psi\left(a_{1}, \ldots, a_{n}, t_{1}\left(c_{1}, \ldots, c_{k}\right), \ldots, t_{m}\left(c_{1}, \ldots, c_{k}\right)\right) \text { iff } \\
& \quad \vDash \psi\left(a_{1}^{\prime}, \ldots, a_{n}^{\prime}, t_{1}\left(c_{1}, \ldots, c_{k}\right) \ldots, t_{m}\left(c_{1}, \ldots, c_{k}\right)\right) .
\end{aligned}
$$

Hence, if $y=s\left(a_{1}, \ldots, a_{n}\right)$ and $y^{\prime}=s\left(a_{1}^{\prime}, \ldots, a_{n}^{\prime}\right)$ are two elements of $\bar{M}(A)$ with similar representations and $\left(a_{1}, \ldots, a_{n}\right)$ and $\left(a_{1}^{\prime}, \ldots, a_{n}^{\prime}\right)$ equivalent over $C$, then for any $L$-formula $\varphi$

$$
\vDash \varphi\left(y, b_{1}, \ldots, b_{m}\right) \text { iff } \vDash \varphi\left(y^{\prime}, b_{1}, \ldots, b_{m}\right),
$$

that is

$$
\operatorname{tp}(y / B)=\operatorname{tp}\left(y^{\prime} / B\right)
$$

It remains to notice that we have at most $|C|$ equivalence classes of $n$-tuples from $A$ over $C$.

Let for an $a \in A$

$$
c(a)=\min \{c \in C \cup\{\infty\}: a<c\} .
$$

It is immediate from definitions that $a_{1}<\cdots<a_{n}$ and $a_{1}^{\prime}<\cdots<a_{n}^{\prime}$ are equivalent over $C$ iff $c\left(a_{1}\right)=c\left(a_{1}^{\prime}\right), \ldots, c\left(a_{n}\right)=c\left(a_{n}^{\prime}\right)$, hence each equvalence class is determined by an $n$-tuple $\left(c_{1}, \ldots, c_{n}\right)=\left(c\left(a_{1}\right), \ldots, c\left(a_{n}\right)\right)$ of elements of $C \cup\{\infty\}$. 


\section{$6.2 \omega$-stability}

Definition A countable theory $T$ is said to be $\omega$-stable (or totally transcendental) if for any model $M$ of $T$ and any countable subset $A \subseteq M$ the set $S(A)$ of complete 1 -types over $A$ is of cardinality at most $\aleph_{0}$.

Proposition 6.1 $T$ is $\omega$-stable if and only if for any $n$, any model $M$ of $T$ and any countable subset $A \subseteq M$ the set $S^{n}(A)$ of complete $n$-types over $A$ is of cardinality at most $\aleph_{0}$.

Proof It is enough to prove that $\omega$-stability implies that $S^{n}(A)$ is countable for all countable $A$. We prove this by induction on $n$.

For $n=1$ the statement is by $\omega$-stability.

We assume $M$ is a monster-model, that is realises all types over any subset we consider.

For $n>1$, assuming by induction argument that $S^{n-1}(A)$ is countable find a countable $M_{0} \prec M$ such that $A \subseteq M_{0}$ and $M_{0}$ realises all the types in $S^{n-1}(A)$. We can further extend $M_{0} \prec M_{1} \prec M$ so that $M_{1}$ is countable and realises all types of $S^{1}\left(M_{0}\right)$. Then $M_{1}$ realises all the types of $S^{n}(A)$. Indeed, if $\left\langle b_{1}, \ldots, b_{n}\right\rangle$ an $n$-tuple in $M$ then there is a $\left\langle b_{1}^{\prime}, \ldots, b_{n-1}^{\prime}\right\rangle$ in $M_{0}$ such that

$$
\operatorname{tp}\left(b_{1}^{\prime}, \ldots, b_{n-1}^{\prime} / A\right)=\operatorname{tp}\left(b_{1}, \ldots, b_{n-1} / A\right) .
$$

The type $p_{A, b_{1}, \ldots, b_{n-1}}$ of $b_{n}$ over $A \cup\left\{b_{1}, \ldots, b_{n-1}\right\}$ corresponds via swapping the $b_{i}$ for $b_{i}^{\prime}$ to a type $p_{A, b_{1}^{\prime}, \ldots, b_{n-1}^{\prime}}$. The latter is realisable in $M_{1}$, say by $b_{n}^{\prime}$. It follows that $\left\langle b_{1}, \ldots, b_{n}\right\rangle$ and $\left\langle b_{1}^{\prime}, \ldots, b_{n}^{\prime}\right\rangle$ satisfy the same formulas with parameters in $A$, that is they realise the same type of $S^{n}(A)$. Hence every type of $S^{n}(A)$ is realised in a countable $M_{1}$, which implies that $S^{n}(A)$ is countable.

Theorem 6.2 If a countable theory $T$ is categorical in some uncountable cardinality $\kappa$ then $T$ is $\omega$-stable.

Proof We are going to prove a formally stronger statement: for any $M \vDash T$ and any $A \subseteq M$ with card $A<\kappa$ the set $S(A)$ is of cardinality at most card $A+\aleph_{0}$. 
Assume otherwise, that is for some $M$ and $A$ with $\aleph_{0} \leq$ card $A=\lambda<\kappa$

$$
\operatorname{card} S(A)>\lambda \text {. }
$$

By the Compactness Theorem and Lowenheim-Skolem up- and down-theorems we may assume that $M$ realises at least $\lambda^{+}$types of $S(A)$ and card $M=\kappa$. On the other hand by the Ehrenfeucht-Mostowski Theorem there is a model $M^{\prime}$ of $T$ of the same cardinality such that for any $A^{\prime} \subseteq M^{\prime}$ with card $A^{\prime} \leq \lambda$ there are at most $\lambda$ types of $S\left(A^{\prime}\right)$ realised in $M^{\prime}$. Hence $M$ can not be isomorphic to $M^{\prime}$. This contradicts the assumptions on categoricity in $\kappa$.

Exercise 6.3 For any countable $\omega$-stable theory for any infinite cardinal $\kappa$ there is an $\omega$-staturated model $M$ of the theory of cardinality $\kappa$.

In fact, the property that $S(A)$ is at most countable for finite A suffices.

Our next goal is to give a more general definition of Morley rank for $\omega$-stable theories.

For a topological space $X$ the Cantor-Bendixson Derivative $d(X)$ is defined as the set of all the limit points in $X$.

Let, by induction:

$d^{0}(X)=X$

$d^{\alpha+1}(X)=d\left(d^{\alpha}(X)\right)$

$d^{\lambda}(X)=\bigcap_{\alpha<\lambda} d^{\alpha}(X)$ for $\lambda$ limit.

For compact $X, d^{\alpha}(X)$ is compact too. The first $\alpha$ where the process is stabilised, i.e. $d^{\alpha+1}(X)=d^{\alpha}(X)$, is called the Cantor-Bendixson rank of $X$, or $\mathrm{CB}(X)$. For $\alpha=\mathrm{CB}(X), d^{\alpha}(X)$ is empty or perfect (the perfect kernel), that is compact without isolated points. By Cantor's arguments a perfect kernel in a space with countable basis is of cardinality $2^{\aleph_{0}}$.

Thus one easily gets

Cantor-Bendixson's Theorem For a countable compact space

$$
\mathrm{CB}(X)<\aleph_{1}, \quad d^{\mathrm{CB}(X)}(X)=\emptyset \text { and } \mathrm{CB}(X) \text { is a successor ordinal. }
$$


Definition Let now $M$ be an $\omega$-saturated model of an $\omega$-stable theory $T$. For an $n$-variable formula $\varphi(\bar{v})$ with parameters in $M$ let $\mathrm{CB}(\varphi)$ be the Cantor-Bendixson rank of the Stone space

$$
S_{\varphi(M)}^{n}=\left\{p \in S^{n}(M): \varphi \in p\right\} .
$$

Example Let $M$ be a strongly minimal structure (and $T$ its theory). Then $S(M) \backslash d^{1}(S(M))$ consists of all the types determined by a formula $v=a$, $a \in M$ and $d^{1}(S(M))$ consists of the unique type containing $\neg v=a$ for all $a \in M . \mathrm{CB}(v=v)=2$.

Proposition 6.2 Given an $\omega$-stable theory, its $\omega$-saturated model $M$ and a definable subset $U$ of $M^{n}$

$\mathrm{CB}(U) \geq \alpha+2$ iff there exists a countable family $\left\{V_{i}: i \in \mathbb{N}\right\}$ of definable mutually disjoint subsets of $U$ such that $\mathrm{CB}\left(V_{i}\right) \geq \alpha+1$ for each $i$.

Proof Define for a complete type $p \in S^{n}(M)$

$$
\mathrm{CB}(p)=\min \left\{\alpha: p \notin d^{\alpha+1}\left(S^{n}(M)\right)\right\} .
$$

Equivalently, $\operatorname{CB}(p)=\alpha$ iff $p$ is isolated in $d^{\alpha}\left(S^{n}(M)\right)$.

Then $\operatorname{CB}(\varphi)>\alpha+1$ iff $S_{\varphi}^{n}(M) \cap d^{\alpha+1}\left(S^{n}(M)\right) \neq \emptyset$ iff $d^{\alpha}\left(S^{n}(M)\right)$ is infinite. But the countable compact $d^{\alpha}\left(S^{n}(M)\right)$ is infinite iff the set of its isolated points is infinite. Thus, equivalently we can choose some $p_{1}, \ldots, p_{i}, \ldots$ isolated in $d^{\alpha}\left(S^{n}(M)\right)$. Let $\psi_{i}$ for each $i \in \mathbb{N}$ be a formula isolating $p_{i}$ in $d^{\alpha}\left(S^{n}(M)\right)$. Then $d^{\alpha}\left(S_{\psi_{i}}^{n}(M)\right) \neq \emptyset$, that is $\mathrm{CB}\left(\psi_{i}\right) \geq \alpha+1$. We may also assume that $\psi_{i}$ is disjoint from $\psi_{j}$ for distinct $i$ and $j$ by changing to $\psi_{i}^{\prime}=\psi_{i} \& \neg \psi_{1} \& \ldots \& \neg \psi_{i-1}$. Under these assumptions $V_{i}=\psi_{i}(M)$ form a family of disjoint subsets of $U=\varphi(M)$ with $\mathrm{CB}\left(V_{i}\right) \geq \mathrm{CB}\left(p_{i}\right)+1=\alpha+1$. Conversely, such a family of $\psi_{i}$ immediately gives rise to a sequence of $p_{i} \in d^{\alpha}\left(S_{\psi_{i}}^{n}(M)\right)$ which witnesses that $\mathrm{CB}(U)>\alpha+1$. 
Corollary 6.1 For any definable $U$ there is a finite $m$ such that $U$ can not contain more than $m$ disjoint definable subsets of $\mathrm{CB}$-rank equal to $\mathrm{CB}(U)$.

Definition The minimal $m$ in the corollary is called the Morley degree of $U$ and denoted $\operatorname{deg}^{*}(U)$.

A definable $U$ in an $\omega$-saturated $M$ is said to be irreducible if $\operatorname{deg}^{*} U=1$.

Definition In an $\omega$-saturated model of an $\omega$-stable theory the Morley rank of definable subset $U \subseteq M^{n}$ is defined as

$$
\mathrm{rk}^{*}(U)=\mathrm{CB}(U)-1
$$

or, equivalently, by induction:

$\operatorname{rk}^{*}(U) \geq 0$ iff $U \neq \emptyset$

$\operatorname{rk}^{*}(U) \geq \alpha$ iff for any $\beta<\alpha$ there are infinitely many disjoint definable subsets of Morley rank greater or equal to $\beta$.

Lemma 6.2.1 Under the assumptions of the definition above assume also that $\mathrm{rk}^{*} U$ is finite and $f: U \rightarrow M^{m}$ is a definable function. Then $\operatorname{rk}^{*} f(U)$ is finite and

$$
\mathrm{rk}^{*} f(U)+\min \left\{\mathrm{rk}^{*} f^{-1}(a): a \in f(U)\right\} \leq \mathrm{rk}^{*} U
$$

Proof Induction on $\operatorname{rk}^{*} f(U)=\gamma$.

For $\gamma=0$ the inequality is obvious. For $\gamma>\beta \geq 0$ we have by definition infinitely many disjoint definable subsets $V_{i} \subseteq f(U)$ of Morley rank greater or equal to $\beta$. Hence, by induction hypothesis

$$
\beta+\min \left\{\mathrm{rk}^{*} f^{-1}(a): a \in V_{i}\right\} \leq \mathrm{rk}^{*} f^{-1}\left(V_{i}\right)
$$

Since $f^{-1}\left(V_{i}\right)$ is a family of disjoint subsets of, again by definition

$$
\gamma+\min \left\{\mathrm{rk}^{*} f^{-1}(a): a \in f(U)\right\} \leq \mathrm{rk}^{*} U
$$


Lemma 6.2.2 Assuming that $M$ is w-saturated, $U$ definable in $M$ and $f$ : $U \rightarrow M^{n}$ definable function with finite fibers $f^{-1}(a)$,

$$
\mathrm{rk}^{*} f(U)=\mathrm{rk}^{*} U \text {. }
$$

Proof Induction on $\mathrm{rk}^{*} U=\gamma$. It is obvious for $\gamma=0$.

By assumptions $f^{-1}(a)$ is finite for any $a \in f(U)$, and by saturatedness we have a common bound: $\left|f^{-1}(a)\right| \leq m$ for all $a \in f(U)$.

Suppose $\gamma>\alpha$. Let $\left\{V_{i}: i \in \mathbb{N}\right\}$ be a countable disjoint family of definable subsets of $U$ of rank $\alpha$. We may assume that each $V_{i}$ is irreducible. Then $\left\{f\left(V_{i}\right): i \in \mathbb{N}\right\}$ is a family of irreducible subsets of rank $\alpha$ (by inductive hypothesis) with the property that an $a \in f(U)$ can be a common point of at most $m$ of the sets of the family. It follows that for a given $i_{0}$ there are at most $m$ distinct $i \in \mathbb{N}$ such that $\mathrm{rk}^{*}\left(f\left(V_{i_{0}}\right) \cap f\left(V_{i}\right)\right)=\alpha$. By removing those $V_{i}$ s we can get an infinite disjoint subfamily $\left\{f\left(V_{i}\right): i \in I\right\}$ of subsets of rank $\alpha$. This witnesses that $\operatorname{rk}^{*} f(U)>\alpha$ and thus $\operatorname{rk}^{*} f(U) \geq \operatorname{rk}^{*} U$. Combining with Lemma 6.2.1 we get the equality.

The following statement establishes the link between $\omega$-stability and the theory of strongly minimal sets.

Proposition 6.3 Let $M$ be an $\omega$-saturated model of an $\omega$-stable theory and $S$ its infinite definable subset. Then there exists a definable set $U \subset S$ of Morley rank 1, irreducible. Such an $U$ as a structure with relation induced from $M$ is strongly minimal.

Proof By assumptions rk $S \geq 1$. Let $U$ be a definable subset of $S$ with minimal positive Morley rank and irreducible. Then by definition rk $U=1$. Also, any partition of $U$ into two definable subsets, $U=U_{1} \dot{U} U_{2}$, implies that rk $U_{1}=0$ or rk $U_{2}=0$, thus one of the sets has to be finite. This imples that $U$ is minimal as a set in $M$ and also as a substructure with the induced relations. Since $M$ is saturated, the pregeometry of $U$ is of infinite dimension. Hence $U$ is strongly minimal.

As a corollary of lemmas above we also have 
Proposition 6.4 If $U \subseteq M^{n}$ is strongly minimal, then $\operatorname{rk}^{*} U^{n}=n$ and for any definable subset $T \subseteq U^{k}$

$$
\mathrm{rk}^{*} T=\mathrm{rk} T
$$

Proof Suppose $T \subseteq U^{k}$ is defined over a finite $A \subseteq M$, and let $\left\langle u_{1}, \ldots, u_{k}\right\rangle \in$ $T$ be a tuple of a maximal dimension, say equal to $r$, over $A$. That is, up to the numeration of variables, $\left\{u_{1}, \ldots, u_{r}\right\}$ is independent over $A$ and $u_{r+1}, \ldots, u_{k} \in \operatorname{acl}\left(A \cup\left\{u_{1}, \ldots, u_{r}\right\}\right.$. Then $r=\operatorname{rk} T$, the Morley rank of section ??. We may assume that $T$ is irreducible and that $x_{r+1}, \ldots, x_{k} \in$ $\operatorname{acl}\left(A \cup\left\{x_{1}, \ldots, x_{r}\right\}\right.$ for all $\left\langle x_{1}, \ldots, u_{k}\right\rangle \in T$.

The projection pr : $U^{k} \rightarrow U^{r}$ along the last $k-r$ coordinates sends $T$ to a subset $\operatorname{pr} T \subseteq U^{r}$ and the fibers of the projection are finite. Hence $\mathrm{rk}^{*} T=\mathrm{rk}^{*} \operatorname{pr} T$. If $r<k$ then by induction we have $\mathrm{rk}^{*} \operatorname{pr} T=\operatorname{rk} T=r$.

If $r=k, \operatorname{rk}^{*} T=\gamma>\alpha$ let $\left\{V_{i}: i \in \mathbb{N}\right\}$ be a disjoint family of subsets of $T$ of rank $\alpha$. Let $A_{i} \supseteq A$ be a finite set of parameters for $V_{i}$ and $v_{i} \in V_{i}$ of maximal dimension over $A_{i}$. By irreducibility, for all $i$, except for maybe one, $\mathrm{rk} V_{i}=\mathrm{rk}\left(v_{i} / A_{i}\right)<r$. It follows by the argument above that $\alpha=\mathrm{rk}^{*} V_{i}=\operatorname{rk} V_{i}<r$. Hence, by definition, $\gamma=\mathrm{rk}^{*} T \leq r$. The converse ineqality is obvious from the fact that $U^{r}$ has infinitely many definable subsets of the form $\{a\} \times U^{r-1}, a \in U$, each of them, by induction, of rank $r-1$.

Hence, $\mathrm{rk}^{*}$ coincides with $\mathrm{rk}$ on strongly minimal subsets and we omit * from now on.

Theorem 6.4 In any uncountably categorical theory Morley rank of any definable set is finite, and all properties of the rank proved for sets definable in strongly minimal structures hold.

Proof In the literature

\section{References}

[CK] C.C.Chang, H.J.Kiesler, Model Theory, North Holland, Amsterdam, 1973 
[Se] A.Seidenberg, Lectures in Projective Geometry, D.van Nostrand Co., 1962

[Z] B.Zilber, Uncountably Categorical Theories AMS Translations of Mathematical Monographs, v.117, Providence, R.I. 1993 